\title{
On Turing machines, groupoids, and Atiyah problem
}

\author{
Dissertation \\ zur Erlangung des mathematisch-naturwissenschaftlichen Doktorgrades \\ "Doctor rerum naturalium" \\ der Georg-August-Universität Göttingen
}

vorgelegt von

Łukasz Grabowski

aus Szczecin

Göttingen 2011 
Referent: Prof. Dr. Andreas Thom

Koreferent: Prof. Dr. Thomas Schick

Tag der mündlichen Prüfung: 
To my family. 


\section{Contents}

1. Introduction 5

A. Results 6

$\begin{array}{ll}\text { B. Summary } & 7\end{array}$

C. Open questions 11

D. Thanks and acknowledgments 12

E. Basic notation and conventions 12

2. Groupoids 13

A. Definitions 13

$\begin{array}{ll}\text { B. Subgroupoids } & 15\end{array}$

$\begin{array}{ll}\text { C. Elements of the groupoid ring as direct integrals of operators } & 17\end{array}$

D. Relation groupoids with finite orbits 18

3. Examples 21

A. Action groupoids and Pontryagin duality 21

B. Computation of Grigorchuk \& Żuk 22

C. Percolation theory, theorem of Lehner, Neuhauser \& Woess 23

4. Turing dynamical systems 25

A. Definitions and basic properties 25

B. Expressing the fundamental values as von Neumann dimensions 28

5. Turing dynamical systems - examples 30

A. Turing dynamical system associated to a set of natural numbers 30

B. Properties of the system $\left(X, T_{X}\right) \quad 32$

C. A "read only" system with irrational fundamental values 35

D. Properties of the system $\left(Y, T_{Y}\right) \quad 36$

6. Atiyah problem 38

A. Preliminaries 38

B. The lamplighter group 40

C. Finitely generated groups 40

D. Finitely presented groups 41

$\begin{array}{ll}\text { References } & 42\end{array}$ 


\section{INTRODUCTION}

The main theorems of the thesis concern studying countable discrete groups through so called $l^{2}$-Betti numbers. These are certain invariants, originally introduced by M. Atiyah in [Ati76] to study free cocompact actions of discrete groups on manifolds. Subsequently, they were studied and used in many different context in geometry and group theory (e.g. [Dod77], [CG86], [Gab02]).

A particular question Atiyah asked in [Ati76] was whether $l^{2}$-Betti numbers can be irrational. Since then, various statements about restrictions on possible values of $l^{2}$-Betti numbers bear the name the Atiyah conjecture (e.g. [DLM $\left.{ }^{+} 03\right]$ ). We depart somewhat from this tradition. Given a countable discrete group $G$, the following question will be referred to as the Atiyah problem for $G$.

Question. What is the set of $l^{2}$-Betti numbers arising from $G$ ?

Let us right away introduce the notation $\mathcal{C}(G)$ for the set in the above question. Over time it has been realized (see [Eck00] and [GLSŻ00]) that $l^{2}$-Betti numbers arising from a given group can be defined purely in terms of $G$, without mentioning manifolds. Consequently, the Atiyah problem can also be phrased purely in terms of $G$. This is the approach we adopt in the thesis and which we now briefly present.

The rational group ring $\mathbb{Q} G$ acts on the Hilbert space $l^{2} G$ by convolution, and similarly matrices $M_{k}(\mathbb{Q} G) \cong M_{k}(\mathbb{Q}) \otimes \mathbb{Q} G$ act on $\left(l^{2} G\right)^{k}$. We have a trace $\tau_{G}$ on $\mathbb{Q} G$ defined by $\tau_{G}(T):=\left\langle T \zeta_{e}, \zeta_{e}\right\rangle$, where $\zeta_{e} \in l^{2} G$ is the vector corresponding to the neutral element of $G$, and we have the induced trace $\operatorname{tr} \otimes \tau_{G}$ on $M_{k}(\mathbb{Q} G)$, also denoted by $\tau_{G}$.

Recall that when $R$ is a ${ }^{*}$-ring of operators on a Hilbert space, together with a trace $\tau$ which is normal (i.e., extends in a continuous way to the weak closure of $R$ ), positive (i.e., $\tau\left(T^{*} T\right) \geq 0$ ) and faithful (i.e., $\tau\left(T^{*} T\right)=0$ implies $T=0$ ), then for a self-adjoint $T \in R$ we can compose the usual projection-valued spectral measure with $\tau$, to obtain the (scalar-valued) spectral measure of $T$. In particular, spectral measure of the set $\{0\}$ is called the von Neumann dimension of the kernel of $T$, and is denoted by $\operatorname{dim}_{\mathrm{vN}} \operatorname{ker} T$. For a non-self-adjoint $T$, one defines $\operatorname{dim}_{\mathrm{vN}} \operatorname{ker} T:=\operatorname{dim}_{\mathrm{vN}} \operatorname{ker} T^{*} T$.

It turns out that $\tau_{G}$ is a positive faithful normal trace on $M_{k}(\mathbb{Q} G)$ and thus we have a von Neumann dimension. A real number $r$ is said to be an $l^{2}$-Betti number arising from $G$ if and only if there exists a matrix $T \in M_{k}(\mathbb{Q} G)$ such that $\operatorname{dim}_{\mathrm{vN}} \operatorname{ker} T=r$.

Much is known about the Atiyah problem for various particular groups. If $G$ is torsion-free, then $\mathcal{C}(G)$ is conjectured to be the set of non-negative integers. This statement is known as the Atiyah conjecture for torsion-free groups (there is a similar conjecture for groups whose torsion subgroups have bounded orders). Cases for which the Atiyah conjecture is known include elementary amenable groups, free groups (see [Lin93] for both classes) and braid groups (see [LS07]). Many results follow by applying versions of Lück's approximation theorem (see [Lüc94], $\left[\mathrm{DLM}^{+} 03\right]$, [Tho08]) to already established results. Perhaps the most familiar consequence of the Atiyah conjecture is the zero divisors conjecture for torsionfree groups. For other results see [Lüc02], Chapter 10. 
Before the work of R. Grigorchuk and A. Żuk in [GŻ01], it had been conjectured that $\mathcal{C}(G) \subset \mathbb{Z}\left(\frac{1}{a_{1}}, \frac{1}{a_{2}}, \ldots\right)$, where $a_{i}$ are orders of torsion subgroups in $G$. However, in [GŻ01] the authors showed that $\operatorname{dim}_{\mathrm{vN}} \operatorname{ker} T=\frac{1}{3}$ for a certain operator $T$ from the group ring of the lamplighter group $\mathbb{Z} / 2 \mathbb{Z} \imath \mathbb{Z}$. Recall that the latter group is a semi-direct product of $\mathbb{Z} / 2 \mathbb{Z}{ }^{\oplus \mathbb{Z}}$ with $\mathbb{Z}$ with respect to the shift action of $\mathbb{Z}$ on $\mathbb{Z} / 2 \mathbb{Z}^{\oplus \mathbb{Z}}$. In particular torsion subgroups of the lamplighter group have orders which are powers of 2 .

Shortly afterwards W. Dicks and T. Schick described in [DS02] an operator T from the group ring of $(\mathbb{Z} / 2 \mathbb{Z} \imath \mathbb{Z})^{2}$ and an heuristic evidence on why $\operatorname{dim}_{\mathrm{vN}} \operatorname{ker} T$ is probably irrational. Nonetheless, the question of irrationality of that specific number has remained open.

A breakthrough came in 2009, when T. Austin showed the following theorem.

Theorem ([Aus09]). The set of $l^{2}$-Betti numbers arising from finitely generated groups is uncountable.

In particular there exist irrational $l^{2}$-Betti numbers. However, [Aus09] did not provide a particular group which gives rise to irrational $l^{2}$-Betti numbers.

\section{1-A. Results}

1.1. Theorem. The set of $l^{2}$-Betti numbers arising from the group $(\mathbb{Z} / 2 \mathbb{Z} \backslash \mathbb{Z})^{3}$ contains

$$
\frac{1}{64}-\frac{1}{8} \sum_{k=1}^{\infty} \frac{1}{2^{k^{2}+4 k+6}}
$$

which is an irrational number.

Irrationality of the number above follows from the fact that its binary expansion is non-periodic. The author does not know whether the current transcendence results cover this number.

It is of some theoretical interest to have explicit finitely presented examples, so we point out also the following corollary.

1.2. Corollary. Let $G$ be a group given by the presentation

$$
\left\langle a, t, s \mid a^{2}=1,[t, s]=1,\left[t^{-1} a t, a\right]=1, s^{-1} a s=a t^{-1} a t\right\rangle .
$$

The set of $l^{2}$-Betti numbers arising from $G^{3}$ contains

$$
\frac{1}{64}-\frac{1}{8} \sum_{k=1}^{\infty} \frac{1}{2^{k^{2}+4 k+6}}
$$

In both Theorem 1.1 and Corollary 1.2, the appropriate matrix whose kernel dimension is as stated can be explicitly written down in terms of the standard generators.

1.3. Theorem. The set of $l^{2}$-Betti numbers arising from finitely generated groups is equal to the set of non-negative real numbers.

The group which realizes a given real number $r$ is "as explicit as the binary expansion of $r^{\prime \prime}$.

We can also say something about the set of $l^{2}$-Betti numbers arising from finitely presented groups. Recall that a set $\Sigma$ of natural numbers is called computable 
if there exists a Turing machine which lists elements of $\Sigma$ in the increasing order (in other words, there exists an algorithm which allows to compute subsequent elements of $\Sigma$ ).

We say that a real number $r$ has computable binary expansion if the fractional part of $r$ is of the form

for some computable set $\Sigma$.

$$
\sum_{i \in \Sigma} \frac{1}{2^{i}}
$$

1.4. Theorem. The set of $l^{2}$-Betti numbers arising from finitely presented groups contains all numbers with computable binary expansions.

Examples of numbers with computable binary expansion include all algebraic numbers, $\pi$ and $e$. A fairly well-known example of a number whose binary expansion is not computable is Chaitin's constant encoding the halting problem (see [CC10]).

Theorem 1.3 has been independently proven by M. Pichot, T. Schick and A. Żuk in [PS Z10]. They also proved a result similar to Theorem 1.4. Let us also mention two later developments: (1) In [LW10], F. Lehner and S. Wagner show that $\mathcal{C}\left(\mathbb{Z} / p \mathbb{Z} \imath F_{d}\right)$ contains irrational algebraic numbers, where $F_{d}$ is the free group on $d$ generators, $d>2, p \geq 2 d-1$; (2) In [Gra10] the present author shows that $\mathcal{C}(\mathbb{Z} / p \mathbb{Z} \imath \Gamma)$ contains transcendental numbers, for all $p>1$ and all groups $\Gamma$ which contain an element of infinite order.

\section{1-B. Summary}

Groupoids. Section 2 introduces a computational tool - measured groupoids, i.e. small categories in which morphisms are all invertible, in which the space of objects is a probability measure space, and in which the space of all morphisms is a measure space, in a compatible way (for example, given a set of objects $U$, the set of identity morphisms of objects in $U$ should have the same measure as $U$ ). In a groupoid $\mathcal{G}$ the space of morphisms is denoted by $\mathcal{G}$ as well. The space objects is denoted by $\mathcal{G}_{0}$.

A measurable edge is a pair $(U, \phi)$, where $U$ is a set of objects and $\phi: U \rightarrow \mathcal{G}$ is a (measurable) choice of one morphism for each object of $U$, such that morphisms corresponding to different objects have different codomains. Each measurable edge gives rise to a convolution operator on $L^{2} \mathcal{G}$, in largely the same way as the group ring $\mathbb{C} G$ of a group $G$ acts on $l^{2} G$. Each function $f \in L^{\infty}\left(\mathcal{G}_{0}\right)$ also acts on $L^{2} \mathcal{G}$, by pointwise multiplication by $f$ composed with the codomain map $\mathcal{G} \rightarrow \mathcal{G}_{0}$. The groupoid ring $\mathbb{C G}$ is defined to be the ring of bounded operators on $L^{2} \mathcal{G}$ generated by measurable edges and $L^{\infty}\left(\mathcal{G}_{0}\right)$.

There is a positive normal faithful trace on $\mathbb{C G}$ which, as explained above, allows to compute von Neumann dimensions of kernels of elements of $\mathbb{C G}$.

A connected component in $\mathcal{G}$ is a maximal set of objects such that between any two objects there is a morphism in $\mathcal{G}$ between them. If a groupoid $\mathcal{G}$ has only finite connected components then computing $\operatorname{dim}_{\mathrm{vN}} \operatorname{ker} T$ reduces to computing the standard dimension of kernels of "T restricted to different connected components". This is made precise in Corollary 2.10, which is the main computational tool. 
Given $T \in \mathbb{C} \mathcal{G}$ we have

$$
\operatorname{dim}_{\mathrm{vN}} \operatorname{ker} T=\sum_{[g]} \frac{\mu([g])}{|V(g)|} \operatorname{dim} \operatorname{ker} T_{g},
$$

where $[g]$ are different isomorphism classes of connected components, $\mu([g])$ is the probability with which given connected component $[g]$ appears in $\mathcal{G}_{0},|V(g)|$ is the number of points in $g$ and $T_{g}$ is the "restriction of $T$ to $g$ ".

Pontryagin duality and action groupoids. Section 3 explains how to pass from certain groups to groupoids. This is done in order to simplify the computations of spectral measures. If $X$ is a compact abelian group and $\rho: \Gamma \curvearrowright X$ is an action of a discrete group $\Gamma$ by group automorphisms, then we also have the dual action $\rho: \Gamma \curvearrowright \widehat{X}$ on the Pontryagin dual of $X$.

On the one hand, we can form the action groupoid $\mathcal{G}(\rho)$ of $\rho$ whose space of objects is $X$ and morphisms whose source is $x \in X$ are elements $(x, \gamma), \gamma \in \Gamma$. Range of a morphism $(x, \gamma)$ is $\rho(\gamma)(x)$, and the composition is the obvious one.

On the other hand we can form the semi-direct product $\widehat{X} \rtimes \Gamma$ and its group $\operatorname{ring} \mathbb{Q}(\widehat{X} \rtimes \Gamma)$.

Pontryagin duality gives us a map $P: \widehat{X} \rightarrow L^{\infty}(X)$. Also, given $\gamma \in \Gamma$, we obtain a measurable edge $x \mapsto(x, \gamma)$ with the domain of definition being all of $X$. Those two maps combine to give a map

$$
P \otimes 1: \mathbb{Q}(\widehat{X} \rtimes \Gamma) \rightarrow \mathbb{C} \mathcal{G}(\rho),
$$

which has the property that $\operatorname{dim}_{\mathrm{vN}} \operatorname{ker} T=\operatorname{dim}_{\mathrm{vN}} \operatorname{ker}(P \otimes 1)(T)$ (Proposition 3.1).

The reason why computations on the right hand side are sometimes easier is as follows: it might be that $\operatorname{supp}(T)$ (the set of elements of $\widehat{X} \rtimes \Gamma$ on which $T$ is supported) generates the whole group; but $\operatorname{supp}(P \otimes 1(T))$ generates a groupoid with finite connected components, and therefore formula (1.1) can be used to compute $\operatorname{dim}_{\mathrm{vN}} \operatorname{ker}(P \otimes 1)(T)$.

Example computations. As an example, we recover in Section 3-B a computation of R. Grigorchuk and A. Żuk from [GŻ01]. Consider the operator $T=$ $t+t^{-1}+t g+g t^{-1}$ in the group ring of $\mathbb{Z} / 2 \mathbb{Z} \imath \mathbb{Z}$, where $t$ generates $\mathbb{Z}$ and $g$ generates $\mathbb{Z} / 2 \mathbb{Z}$. It is an example of an operator whose support generates the whole group $\mathbb{Z} / 2 \mathbb{Z} \succ \mathbb{Z}$, yet its image $(P \otimes 1)(T)$ in the groupoid ring of the Bernoulli action $\mathbb{Z} \curvearrowright \mathbb{Z} / 2 \mathbb{Z}^{\mathbb{Z}}$ has support generating a subgroupoid with finite connected components. Therefore, we can use (1.1) to show that $\operatorname{dim}_{\mathrm{vN}} \operatorname{ker} T=\frac{1}{3}$.

As another example, in Section 3-C we derive a generalization, proved originally by F.Lehner, M. Neuhauser and W. Woess in [LNW08], of the above computation to arbitrary wreath products $\mathbb{Z} / p \mathbb{Z}<\Gamma$.

Logic of the proofs. By now we can explain the strategy to prove, say, Theorem 1.3. For a given real number $r$ find a groupoid $\mathcal{G}_{r}$, which is an action groupoid for an action $\rho_{r}: \Gamma \curvearrowright X$ (note that $\Gamma$ and $X$ do not depend on $r$ ) and an operator $T_{r} \in \mathbb{C G}_{r}$ whose support generates a subgroupoid with finite connected components. Then use formula (1.1) to show that $\operatorname{dim}_{\mathrm{vN}} \operatorname{ker} T_{r}=r$, and check that $T_{r}$ is in the image of the map $P \otimes 1: \mathbb{Q}(\widehat{X} \rtimes \Gamma) \rightarrow \mathbb{C} \mathcal{G}_{r}$. This shows that $r$ is an $l^{2}$-Betti number arising from $\widehat{X} \rtimes \Gamma$. 
Turing dynamical systems. The question is, where to get the groupoid $\mathcal{G}_{r}$ from. For this we introduce in Section 4 Turing dynamical systems. They essentially consist of (1) an action $\rho: \Gamma \curvearrowright X,(2)$ a map $T_{X}: X \rightarrow X$ defined by restrictions of elements of $\Gamma$ to a finite number of subsets of $X$, and (3) three disjoint distinguished subsets $I, A$ and $R$ of $X$, referred to as the initial, accepting and rejecting sets. Such a Turing dynamical system is denoted by $\left(X, T_{X}\right)$.

Given $\left(X, T_{X}\right)$ we get two groupoids. The first one is simply $\mathcal{G}(\rho)$, the action groupoid of $\rho$. For the second, note that $T_{X}$ gives a structure of a directed graph on $X$ : there is an edge from $x$ to $y$ if $T_{X}(x)=y$. The second groupoid, $\mathcal{G}\left(T_{X}\right)$, has $X$ as the space of objects, and morphisms are generated by the directed graph just described. Although it is not entirely clear from the description we gave, $\mathcal{G}\left(T_{X}\right)$ is a subgroupoid of $\mathcal{G}(\rho)$.

In Section 4 -B we describe an operator $S \in \mathbb{C G}\left(T_{X}\right)$ and prove the following (Theorem 4.11).

Theorem. If in the graph induced by $T_{X}$ each connected component (1) has a final point (i.e. a point without outgoing edges) in $A \cup R$, and (2) has at most one point in $I$, then

$$
\operatorname{dim}_{v N} \operatorname{ker} S=\mu(I)-\mu\left(\left\{x \in I: T_{X}^{k}(x) \in A \text { for some } k\right\}\right) .
$$

Furthermore, because of a general condition in Lemma 3.2, we see that $S$ is in the image of $P \otimes 1$. Therefore, in order to prove that a given number $r$ is an $l^{2}$-Betti number arising from some group, it is enough to find a Turing dynamical system such that $\mu(I)-\mu\left(\left\{x \in I: T_{X}^{k}(x) \in A\right.\right.$ for some $\left.\left.k\right\}\right)=r$ (and check that conditions (1) and (2) hold). This is done in Section 5.

The proof of the theorem above is an application of the formula (1.1), which can be used, since (1) implies that the connected components of $\mathcal{G}\left(T_{X}\right)$ are finite.

We finish the summary by informally explaining what is a Turing machine, how does it give rise to a Turing dynamical system, and what is the meaning of $\mu\left(\left\{x \in I: T_{X}^{k}(x) \in A\right.\right.$ for some $\left.\left.k\right\}\right)$ for such systems. The examples from Section 5 are (almost) obtained by performing the descibed construction for particular Turing machines. Although we do not use Turing machines per se in the main body of the thesis, reading Section 5 is probably easier when the reader has some intuition about them.

Turing machines. Let $S$ be a finite set of states and $M$ be a finite set of symbols. A Turing machine whose set of states is $S$ and which operates on the alphabet $M$ consists of four components; three of them can be thought of as a hardware: an infinite tape with symbols written on it, a tape reader, and a state register. The fourth component, a transition table, should be thought of as a software.

The transition table consists of a single entry of the type:

"If the current state is $\sigma$ and the symbol under the tape reader is $m$, then do ..." for each pair $(m, \sigma) \in M \times S$.

We assume that the tape have infinitely many symbols written on it, i.e. there are no empty cells on the tape. We assume that three elements of $S$ are distinguished: Initial, Rejecting, and Accepting states (also denoted by $i, r$ and $a$ ). It is 
assumed that the Turing machine always starts its operation in the Initial state, and it stops to operate whenever it reaches either the Rejecting or the Accepting state.

At any given moment the machine is in one of the states. In a single step of operation it does the following things: it reads a symbol on the tape under the tape reader, and then it performs a subset of the following operations, depending on what is written after "do" in the appropriate entry of the transition table:

(1) it changes the symbol on the tape under the tape reader into some other symbol.

(2) it shifts the tape reader to the right or to the left

(3) it changes the state it is currently in into some other state

Associated Turing dynamical systems. Given a Turing machine whose set of symbols is $M$ and whose set of states is $S$, we associate to it a Turing dynamical system as follows. The measure space is "the space of configurations of the Turing machine". i.e. $X:=M^{\mathbb{Z}} \times S$ (with the product measure), where $M^{\mathbb{Z}}$ is the product of infinitely many copies of $M$ indexed by $\mathbb{Z}$; elements of it should be thought of as infinite tapes.

Let $\Gamma$ be the group of measure-preserving automorphisms of $X$ generated by the shift on $M^{\mathbb{Z}}$, bijections of $S$ and automorphisms $K_{\beta}$ of $M^{\mathbb{Z}}$, for every bijection $\beta$ of $M$, defined as

$$
K_{\beta}\left(\left(m_{i}\right)\right)_{j}= \begin{cases}m_{j} & \text { if } j \neq 0 \\ \beta\left(m_{0}\right) & \text { if } j=0\end{cases}
$$

In this way we obtain an action $\rho: \Gamma \curvearrowright X$.

The crucial observation is that the operations (1), (2) and (3) above correspond to elements of $\Gamma$. More precisely, the set $X$ can be divided into a finite number of subsets $[\underline{m}][\sigma], m \in M, \sigma \in S$ :

$$
[\underline{m}][\sigma]:=\left\{\left(\left(m_{i}, \tau\right): m_{0}=m, \sigma=\tau\right\},\right.
$$

and for every set $[\underline{m}][\sigma]$ we have an element $\gamma(m, \sigma) \in \Gamma$ corresponding to the entry in the transition table which describes the behaviour of the Turing machine for the symbol $m$ and the state $\sigma$. Now, we can define the map $T_{X}: X \rightarrow X$ as

$$
T_{X}(x):=\gamma(m, \sigma)(x) \quad \text { for } x \in[\underline{m}][\sigma] .
$$

It should be clear that if we have a configuration $x \in X$ of our Turing machine then $T_{X}(x)$ is the configuration after a single step of operation of this Turing machine.

The Initial, Accepting and Rejecting sets are defined as

$$
I=\bigcup_{m \in M}[\underline{m}][i], \quad A=\bigcup_{m \in M}[\underline{m}][a], \quad R=\bigcup_{m \in M}[\underline{m}][r] .
$$

Let us now give a more concrete example to understand the set $\left\{x \in I: T_{X}^{k}(x) \in\right.$ $A$ for some $k\}$ (called the first fundamental set of $\left(X, T_{X}\right)$ ), and its measure (called the first fundamental value of $\left(X, T_{X}\right)$ ).

Let $P$ be a Turing machine which "computes the digits of $\pi^{\text {" }}$ in the following sense. $P$ operates on the symbols $A, B, C, D$. Denote the symbol which is under the tape reader before the machine starts to operate by $m_{0}$, the symbol immediately to its right by $m_{1}$, and so on. Suppose $P$ is put into the initial state and given a tape for which $m_{0}, m_{1}, \ldots, m_{k} \in\{A, B\}, m_{k+1} \in\{C, D\}$. Then $P$ ends 
in the accepting state if $k$ is such that the $k^{\prime}$ th digit in the binary expansion of the fractional part of $\pi$ is 1 . Otherwise, it ends in the Rejecting state or operates forever.

From the description of $P$ it follows that the first fundamental set is the union

$$
\bigcup_{k \in \Sigma} F_{k}
$$

where $\Sigma$ is the set of those natural numbers $N$ such that the binary expansion of the fractional part of $\pi$ has 1 at $N^{\prime}$ th place, and $F_{k}$ is defined as

$$
F_{k}=\left\{\left(\left(m_{j}\right), \sigma\right): m_{0}, m_{1}, \ldots, m_{k} \in\{A, B\}, m_{k+1} \in\{C, D\}, \sigma=i\right\} .
$$

Note that

$$
\mu\left(F_{k}\right)=\frac{1}{|S|} \cdot \frac{1}{2^{k+2}} .
$$

Accordingly, the fundamental value is equal to $\frac{1}{4|S|} \cdot\{\pi\}$, where $\{\pi\}$ is the fractional part of $\pi$.

Note however that we can not use the Turing dynamical system obtained from the machine $P$ to prove that $\frac{1}{4|S|} \cdot\{\pi\}$ is an $l^{2}$-Betti number arising from some group. There are two reasons. First, the measure space $X$ of configurations of $P$ is not a compact topological group, so we do not have a map $P \otimes 1: \mathbb{Q}(\widehat{X} \rtimes \Gamma) \rightarrow \mathbb{C G}(\rho)$, because $\widehat{X}$ does not exist. However, this is easily repairable.

But second, we did not give any details on how $P$ operates precisely, and so it is not possible to check conditions (1) and (2) to apply the formula (1.2). This is a serious obstruction and the reason why examples in Section 5 are much more explicit, so that the conditions (1) and (2) can actually be checked.

\section{1-C. Open questions}

All questions are well-known to the experts.

Question 1. What is the set of $l^{2}$-Betti numbers arising from finitely presented groups?

Note that the set in question is countable. In [PS Z10] different numbers appear than those covered by Theorem 1.4. On the other hand, note that every $l^{2}$-Betti number is, by functional calculus, a limit of a sequence $\tau_{G}\left((1-T)^{n}\right)$, where $T \in$ $M_{k}(\mathbb{Q} G)$ and $\tau_{G}$ is the group trace. If the group $G$ is sofic, then there are bounds known on what $n$ one has to take to be $\varepsilon$-close to the limit (this follows from the determinant conjecture, see [ES05]). It follows that if $G$ is a sofic group, then elements of $\mathcal{C}(G)$ are computable by a Turing machine with an oracle for the word problem of $G$. If $G$ is finitely presented then the word problem of $G$ is known to be not harder than the halting problem. This gives some bound on what $l^{2}$-Betti numbers can arise from (sofic) finitely presented groups; however this bound seems to be far away for the techniques presented here or in [PS Z10].

Question 2. For a group $G$ and a ring $k \subset \mathbb{C}$, define $\mathcal{C}(G, k)$ to be the set of those $r$ such that there exists $T \in k G$ with $\operatorname{dim}_{v N} \operatorname{ker} T=r$. By definition, $\mathcal{C}(G, \mathbb{Q})=\mathcal{C}(G)$. Is it true that for every group $G$ we have $\mathcal{C}(G)=\mathcal{C}(G, \mathbb{C})$ ? In particular, is it true that the set $\mathcal{C}(G, \mathbb{C})$ is countable? 
The answers are trivially yes for those torsion-free groups (or groups with bounded torsion subgroups) for which the so called strong Atiyah conjecture holds: $\mathcal{C}(G, \mathbb{C})=\mathbb{N}$ (this has to be modified appropriately for bounded torsion groups). Examples include free groups and bounded-torsion elementary amenable groups (see [Lin93]). On the other hand, the answers are not known even for $\mathbb{Z} / 2 \mathbb{Z} \imath \mathbb{Z}$. This motivates the next question.

Question 3. What is $\mathcal{C}(\mathbb{Z} / 2 \mathbb{Z} \backslash \mathbb{Z})$ ?

In Theorem 1.1 we prove $\mathcal{C}\left((\mathbb{Z} / 2 \mathbb{Z} \backslash \mathbb{Z})^{3}\right) \nsubseteq \mathbb{Q}$. After the first version of this article was submitted to arXiv, F. Lehner and S. Wagner showed in [LW10] that $\mathcal{C}\left(\mathbb{Z} / p \mathbb{Z} \imath F_{d}\right)$ contains irrational algebraic numbers, where $F_{d}$ is the free group on $d$ generators, $d>2, p \geq 2 d-1$, which subsequently led the author to show in [Gra10] that $\mathcal{C}(\mathbb{Z} / p \mathbb{Z}<\mathbb{Z})$ contains transcendental numbers, for all $p>1$. This raises the question whether $\mathcal{C}(\mathbb{Z} / p \mathbb{Z} \imath \mathbb{Z})$ contains irrational algebraic numbers. In fact, $\mathcal{C}\left((\mathbb{Z} / p \mathbb{Z} \succ \mathbb{Z})^{k}\right)$ contains algebraic numbers of degree $k$, as will be shown in a future version of [Gra10].

Question 4. Are $l^{2}$-Betti numbers of a countable discrete group rational?

For a precise definition of $l^{2}$-Betti numbers of a group, see e.g. [Eck00] or [Lüc02] for a more general definition. If $r$ is an $l^{2}$-Betti number of a group $G$, then it is in particular an $l^{2}$-Betti number arising from $G$, but not the other way around. All the examples in the literature so far, of groups which give rise to irrational $l^{2}$-Betti number, have an infinite normal amenable subgroup. This implies that all their $l^{2}$-Betti numbers are 0 (see [Lüc02], Theorem 7.2).

\section{1-D. Thanks and acknowledgments}

This thesis is a continuation of the ideas contained in the papers [GŻ01], [DS02], and [Aus09]. In particular, the main innovation - Turing dynamical systems - was largely motivated by the "pattern recognition" idea of [Aus09]. On the other hand, Section 2 came about as an attempt to understand the proof of Lemma 3.6 from [DS02].

I would like to thank Thomas Schick and Andreas Thom for helpful discussions; in particular for explaining to me the aforementioned "pattern recognition" idea.

I owe special thanks to Andreas Thom, for encouragements to write down early versions of this work, and to Manuel Koehler, for committing many hours to discussing technical details of Section 2.

I have had a great luck to have very critical readers of the draft versions of this thesis. Comments of Robin Deeley, Światosław Gal, Jarek Kędra, Thomas Schick and Andreas Thom greatly improved the exposition, as well as eliminated a number of typos and grammatical mistakes.

I also thank Franz Lehner and Stefaan Vaes for helpful comments.

Finally, parts of the work presented here were completed during my stay at Université de la Méditerranée in Marseille in November 2009. My visit there was arranged thanks to Michael Puschnigg, whom I would like to whole-heartedly thank for this and for his kind hospitality. 


\section{1-E. Basic notation and conventions}

Throughout the thesis the term measurable space refers to a standard Borel space. The word subset means measurable subset, whenever it makes sense. In the proofs, we sometimes do not check that the sets we work with are measurable, but in all the cases such checks are straight-forward.

If $U$ is a subset of $X$ then $\chi_{U}: X \rightarrow\{0,1\}$ and $\chi(U): X \rightarrow\{0,1\}$ denote the characteristic function of $U$ in $X$.

If $S$ is a set then cardinality of $S$ is denoted by $|S|$. The Hilbert space whose orthonormal basis consists of elements of $S$ is denoted by $l^{2}(S)$ or $l^{2} S$. The standard basis vectors are denoted by $\zeta_{s}, s \in S$.

If $g$ is a graph then its sets of vertices and edges are denoted by $V(g)$ and $E(g)$. If $g$ is an oriented graph and $e \in E(g)$, then the starting and ending points of $e$ are denoted by $s(e)$ and $r(e)$ ( $r$ stands for range). The Hilbert space $l^{2}(V(g))$ is denoted also by $l^{2} g$.

A full subgraph of a graph $g$ is a subgraph $h$ with the property that if $e \in E(g)$ is an edge between two vertices in $V(h)$, then $e \in E(h)$.

If $v$ is a vertex in an oriented graph with only outgoing edges, $v$ is called a starting vertex. If $v$ has only incoming edges then it is called a final vertex.

Given a ring $R$, the ring of $k \times k$-matrices over $R$ is denoted by $M_{k}(R)$. A trace $\tau$ on $R$ is a function $\tau: R \rightarrow \mathbb{C}$ such that $\tau(a b)=\tau(b a)$. The standard trace (i.e. sum of diagonal elements) on $M_{k}(\mathbb{C})$ is denoted by tr. If $R$ is a ${ }^{*}$-ring of operators on a Hilbert spaces then we also require that $\tau\left(T^{*} T\right)$ is a non-negative real number, for all $T \in R$. If $R$ is an algebra over a field $\mathbb{F} \subset \mathbb{C}$ and $\tau$ is a trace on $R$, then we have an induced trace on $M_{k}(R) \cong M_{k}(\mathbb{F}) \otimes R$ given by $\operatorname{tr} \otimes \tau$, The induced trace is also denoted by $\tau$.

If $R$ is a ${ }^{*}$-ring of operators on a Hilbert space, then a trace $\tau$ on $R$ is called normal if it extends to a continuous trace on the weak completion of $R$. A trace is faithful if, for every $T, \tau\left(T^{*} T\right)=0$ implies $T=0$. All traces we will consider are faithful and normal.

If $R$ is a *-ring of operators on a Hilbert space, $\tau$ is a faithful normal trace on $R$, and $T^{*}=T \in R$ then the spectral measure of $T$ is the usual projectionvalued spectral measure of $T$ composed with $\tau$ (it makes sense to evaluate $\tau$ on spectral projections of $T$, since the latter are in the weak completion of $R$ ). The spectral measure of the set $\{0\}$ is called von Neumann dimension of kernel of $T$, denoted by $\operatorname{dim}_{\mathrm{vN}} \operatorname{ker}(T)$. For a non-self-adjoint $T$ we $\operatorname{define} \operatorname{dim}_{\mathrm{vN}} \operatorname{ker}(T)=$ $\operatorname{dim}_{\mathrm{vN}} \operatorname{ker}(T)$.

We say that the spectral measure of $T^{*}=T \in R$ is pure-point, or that $T$ has pure-point spectrum, if the spectral measure of $T$ is a countable sum of measures supported on single points.

\section{Groupoids}

\section{2-A. Definitions}

For more detailed information on groupoids see [ST10] and references therein.

A groupoid $\mathcal{G}$ is a small category whose morphisms are all invertible. The set of objects is denoted by $\mathcal{G}_{0}$ and the set of all morphisms is denoted by $\mathcal{G}$. The 
embedding $1: \mathcal{G}_{0} \rightarrow \mathcal{G}$ sends an object $x$ to the identity morphism on $x$. The space $\mathcal{G}_{0}$ will be often identified with a subset of $\mathcal{G}$ via this embedding.

The maps $s, r: \mathcal{G} \rightarrow \mathcal{G}_{0}$, source and range maps, associate to a morphism its domain and codomain. Composition is a map $\mathcal{G}_{r} \times{ }_{s} \mathcal{G} \rightarrow \mathcal{G}$; composition of morphisms $\gamma: x \rightarrow y$ and $\gamma^{\prime}: y \rightarrow z$ is denoted by $\gamma \gamma^{\prime}$. Given $\gamma: x \rightarrow y$, the inverse of $\gamma$ is denoted either by $i(\gamma)$ or by $\gamma^{-1}$.

For $x \in \mathcal{G}_{0}$, the sets $s^{-1}(x)$ and $r^{-1}(x)$ are denoted by $s^{*} x$ and $r^{*} x$. The set of those objects $y$ for which there exists a morphism between $x$ and $y$ is referred to as the orbit of $x$, denoted by $\mathcal{G} x$.

A discrete measurable groupoid is a groupoid together with a structure of a measurable space on $\mathcal{G}$, and such that $\mathcal{G}_{0}$ is a measurable subset, fibers of the maps $s$ and $r$ are countable, and the structure maps $s, r, i$ and composition are measurable.

A discrete measured groupoid is a discrete measurable groupoid $\mathcal{G}$ together with a measure $\mu$ on $\mathcal{G}_{0}$, such that the measures

$$
\mathcal{G} \supset U \mapsto \int_{\mathcal{G}_{0}}\left|r^{-1}(x) \cap U\right| d \mu(x)
$$

and

$$
\mathcal{G} \supset U \mapsto \int_{\mathcal{G}_{0}}\left|s^{-1}(x) \cap U\right| d \mu(x)
$$

are equal. This measure on $\mathcal{G}$ is also denoted by $\mu$.

From now on all groupoids will be discrete measured, unless explicitly stated otherwise. The following lemma is a direct consequence of the definition of measure on $\mathcal{G}$.

2.1. Lemma. Suppose $U \subset \mathcal{G}$ is such that $r$ restricted to $U$ is an injection. Then measure of $U$ in $\mathcal{G}$ is the same as the measure of $r(U)$ in $\mathcal{G}_{0}$.

We say that a groupoid $\mathcal{G}$ is a relation groupoid, if for almost all pairs $(x, y)$ of objects (with respect to the product measure on $\mathcal{G}_{0} \times \mathcal{G}_{0}$ ) there is at most one morphism from $x$ to $y$. If $\mathcal{G}$ is a relation groupoid then we freely use the identification of $s^{*} x$ with $\mathcal{G} x$ given by $s^{*} x \ni \gamma \mapsto r(\gamma)$.

A measurable edge is a pair $(U, \phi)$, where $U \subset \mathcal{G}_{0}$ and $\phi: U \rightarrow \mathcal{G}$, such that $s \circ \phi: U \rightarrow \mathcal{G}_{0}$ is the identity embedding, and $r \circ \phi: U \rightarrow \mathcal{G}_{0}$ is injective. Note that $\phi$ and $r \circ \phi$ are automatically measure preserving. For the most part, we write simply $\phi$, with the understanding that $U=\operatorname{Dom}(\phi)$ is the domain of definition of $\phi$. If $\phi$ is a measurable edge, then $\phi^{-1}$, the inverse of $\phi$, is the measurable edge with $\operatorname{Dom}\left(\phi^{-1}\right)=r(\operatorname{Im}(\phi))$, and such that $\phi^{-1}(r(\phi(x)))=i(\phi(x))$.

2.2. Lemma. There exists a countable family of measurable edges whose images are disjoint and such that the union of all their images is all of $\mathcal{G}$.

Proof. The statement follows from a theorem of Luzin and Novikov (see [Kec95], Theorem 18.10): there exists a division of $\mathcal{G}$ into countably many disjoint sets such that the restriction of $s$ to any of them is injective (this is true for any map with countable fibers between measurable spaces). 
Given a measurable edge $\phi$, define a bounded operator on $L^{2} \mathcal{G}=L^{2}(\mathcal{G}, \mu)$, denoted also by $\phi$, by

$$
\phi(F)(\gamma)= \begin{cases}F\left(\gamma \phi^{-1}(r(\gamma))\right) & \text { if } r(\gamma) \in \operatorname{Dom}\left(\phi^{-1}\right), \\ 0 & \text { otherwise, }\end{cases}
$$

where $F \in L^{2}(\mathcal{G})$.

Given $f \in L^{\infty}\left(\mathcal{G}_{0}\right)$ and $F \in L^{2}(\mathcal{G})$, we define $f(F) \in L^{2}(\mathcal{G})$ to be $f(F)(\alpha):=$ $f(r(\alpha)) F(\alpha)$. This is an action of $L^{\infty}\left(\mathcal{G}_{0}\right)$ on $L^{2}(\mathcal{G})$.

The groupoid ring of $\mathcal{G}$, denoted by $\mathbb{C} \mathcal{G}$, is the ring of bounded operators on $L^{2}(\mathcal{G})$ generated by measurable edges and $L^{\infty}(X)$. We note that $\mathbb{C G}$ is ${ }^{*}$-closed (compare Lemma 2.3 below).

Given a measurable edge $\phi$ and a set $U \subset \operatorname{Dom}(\phi)$, we let $\phi_{\mid U}$ and $\phi_{U}$ denote the restriction of $\phi$ to $U$.

2.3. Lemma. Let $\phi$ be a measurable edge and $f \in L^{\infty}(X)$. Then, in $\mathbb{C} G$, we have

(1) $\phi f=\phi_{\mid \operatorname{supp}(f) \cap \operatorname{Dom}(\phi)} f$,

(2) If $\operatorname{Dom}(\phi) \subset U$, and $\chi$ is the characteristic function of $U$ then $\phi \chi=\phi$.

(3) $\phi^{*}=\phi^{-1}$,

(4) $\phi f=\phi(f) \phi$,

where $\phi(f) \in L^{\infty}\left(\mathcal{G}_{0}\right)$ is defined by the formula

$$
\phi(f)(x)= \begin{cases}f\left(r\left(\phi^{-1}(x)\right)\right) & \text { if } x \in \operatorname{Dom}\left(\phi^{-1}\right), \\ 0 & \text { otherwise. }\end{cases}
$$

Proof. We only prove (4). The other statements are proved similarly and left to the reader as an exercise.

Let $F \in L^{2}(\mathcal{G})$. Then $f(F)(\alpha)=f(r(\alpha)) \cdot F(\alpha)$ and therefore

$$
(\phi f)(F)(\alpha)= \begin{cases}f\left(r\left(\alpha \phi^{-1}(r(\alpha))\right)\right) \cdot F\left(\alpha \phi^{-1}(r(\alpha))\right) & \text { if } r(\alpha) \in \operatorname{Dom}\left(\phi^{-1}\right), \\ 0 & \text { otherwise. }\end{cases}
$$

On the other hand from (2.1) we get

$$
(\phi(f) \phi)(F)(\alpha)= \begin{cases}\phi(f)(r(\alpha)) \cdot F\left(\alpha \phi^{-1}(r(\alpha))\right) & \text { if } r(\alpha) \in \operatorname{Dom}\left(\phi^{-1}\right), \\ 0 & \text { otherwise }\end{cases}
$$

but, from (2.2) we see that, if $r(\alpha) \in \operatorname{Dom}\left(\phi^{-1}\right)$ then $\phi(f)(r(\alpha))=f\left(r\left(\phi^{-1}(r(\alpha))\right)\right)$, and so the claim follows from noting that $r(\alpha \beta)=r(\beta)$ for every composable pair $\alpha, \beta$ of morphisms.

In particular, each element of $\mathbb{C G}$ can be (non-uniquely) represented by a finite linear combination of operators $\phi \cdot f$, where $f \in L^{\infty}\left(\mathcal{G}_{0}\right)$, and $\phi$ is a measurable edge.

The trace $\tau_{\mathcal{G}}$ on $\mathbb{C G}$ is defined by the formula

$$
\tau_{\mathcal{G}}(T):=\left\langle T \chi_{0}, \chi_{0}\right\rangle_{L^{2} \mathcal{G}},
$$

where $\chi_{0}$ is the characteristic function of $\mathcal{G}_{0} \subset \mathcal{G}$. The extension of $\tau_{\mathcal{G}}$ to the weak completion of $\mathcal{G}$ is positive, faithful and normal.

\section{2-B. Subgroupoids}

Let $A=\left(\phi_{i}\right)_{i \in I}$ be a family of measurable edges. The subgroupoid generated by $A$, denoted by $\mathcal{G}(A)$, is the discrete measured groupoid whose space of 
objects is equal to $\mathcal{G}_{0}$ and whose morphisms are generated by $\phi_{i}, \phi_{i}^{-1}$, and identity morphisms. More precisely, morphisms in $\mathcal{G}(A)$ are the subset of $\mathcal{G}$ consisting of (1) those $\gamma \in \mathcal{G}$ such that there exists a finite sequence $\gamma_{1}, \ldots, \gamma_{n}$ such that $\gamma=\gamma_{1} \ldots \gamma_{n}$, and for every $j$ either $\gamma_{j}$ or $\gamma_{j}^{-1}$ is in the image of some $\phi_{i}$, and (2) all identity morphisms.

A subgroupoid of a groupoid is a subgroupoid generated by a family of measurable edges.

2.4. Proposition. Let $\mathcal{H}$ be a subgroupoid of $\mathcal{G}$. Note that if $\phi$ is a measurable edge in $\mathcal{H}$, then it is also a measurable edge in $\mathcal{G}$. Similarly, elements of $L^{\infty} \mathcal{H}_{0}$ are at the same time elements of $L^{\infty} \mathcal{G}_{0}$. These two identifications extend to a trace-preserving ${ }^{*}$-embedding $\mathbb{C H} \hookrightarrow \mathbb{C} \mathcal{H}$.

Proof. Let $T \in \mathcal{H}$ be represented by a finite sum $\sum \phi_{i} f_{i}$. Note that

$$
\begin{aligned}
\tau_{\mathcal{H}}\left(\sum \phi_{i} f_{i}\right)=\sum\left\langle\left(\phi_{i} f_{i}\right)\left(\chi_{0}\right), \chi_{0}\right\rangle_{L^{2} \mathcal{H}} & =\int_{K_{i}} \phi_{i}\left(f_{i}\right) \\
& =\sum\left\langle\left(\phi_{i} f_{i}\right)\left(\chi_{0}\right), \chi_{0}\right\rangle_{L^{2} \mathcal{G}}=\tau_{\mathcal{G}}\left(\sum \phi_{i} f_{i}\right),
\end{aligned}
$$

where $K_{i}=\left\{x \in \mathcal{G}_{0}: \phi_{i}(x)\right.$ is the identity morphism of $\left.x\right\}$.

Therefore, we have a map from the set of finite sums representing elements of $\mathbb{C H}$ to the set of finite sums representing elements of $\mathbb{C G}$, which is trace preserving and ${ }^{*}$-preserving. It follows that this map induces a well-defined linear *-embedding of $\mathbb{C H}$ into $\mathbb{C G}$ because both $\tau_{\mathcal{H}}$ and $\tau_{\mathcal{G}}$ are faithful.

2.5. Corollary. Let $T^{*}=T \in \mathbb{C} G$ be represented by a sum $\sum_{i=1}^{n} f_{i} \cdot \alpha_{i}$, where $f_{i} \in L^{\infty}\left(\mathcal{G}_{0}\right)$, and $\alpha_{i}$ are measurable edges. Let $\mathcal{H}$ be the groupoid generated by the measurable edges $\alpha_{i}$. Then $T$ is in the image of the embedding $\mathbb{C H} \hookrightarrow \mathbb{C G}$, and the corresponding element of $\mathbb{C H}$ is also denoted by $T$. The spectral measure of $T$ in $\mathbb{C H}$ is the same as the spectral measure of $T$ in $\mathbb{C G}$.

This corollary is a special case of the following lemma which will be used several times again.

2.6. Lemma. Let $A$ and $B$ be ${ }^{*}$-rings with traces $\tau_{A}$ and $\tau_{B}$ of operators on the Hilbert spaces $H_{A}$ and $H_{B}$. Moreover, assume that the traces extend to faithful normal traces on the weak completions of $A$ and $B$ in the algebras of bounded operators on $H_{A}$ and $H_{B}$, respectively. Let $\phi: A \rightarrow B$ be a trace preserving ${ }^{*}$ homomorphism and $T^{*}=T \in A$. Then the spectral measure of $T$ with respect to $\tau_{A}$ is the same as the spectral measure of $\phi(T)$ with respect $\tau_{B}$.

Proof. Since $\phi$ is ${ }^{*}$-preserving, $\phi(T)$ is also self-adjoint. The spectral measure, as any $\sigma$-additive measure, is determined by measures of intervals. Let $I$ be an interval and $p_{n}$ be a sequence of polynomials converging to $\chi_{I}$ pointwise, everywhere on $\mathbb{R}$. By the definition of the spectral measure, we need to show $\tau_{A}\left(\chi_{I}(T)\right)=\tau_{B}\left(\chi_{I}(\phi(T))\right.$.

By the spectral theorem, we have $p_{n}(T) \rightarrow \chi_{I}(T)$, and $p_{n}(\phi(T)) \rightarrow \chi_{I}(\phi(T))$. Since $\phi$ is a homomorphism, we have $p_{n}(\phi(T))=\phi\left(p_{n}(T)\right)$. Since $\tau$ is normal, we have $\tau_{A}\left(p_{n}(T)\right) \rightarrow \tau_{A}\left(\chi_{I}(T)\right)$, and $\tau_{B}\left(\phi\left(p_{n}(T)\right)\right) \rightarrow \tau_{B}\left(\chi_{I}(\phi(T))\right.$. The claim follows since $\phi$ is assumed to be trace preserving, in particular $\tau_{B}\left(\phi\left(p_{n}(T)\right)\right)=$ $\tau_{A}\left(p_{n}(T)\right)$. 


\section{2-C. Elements of the groupoid ring as direct integrals of operators}

Given an operator $\sum f_{i} \cdot \phi_{i} \in \mathbb{C G}$ and $x \in \mathcal{G}_{0}$, we want to "restrict" the operator to the space $l^{2}\left(s^{*} x\right)$. To define it precisely, we use direct integrals of fields of Hilbert spaces and of operators. For definitions and notation see [Fol95], Chapter 7.4 .

Unless explicitly stated otherwise, all integrals are taken over the space $\mathcal{G}_{0}$.

Consider the field $x \mapsto l^{2}\left(s^{*} x\right)$ of Hilbert spaces over $\mathcal{G}_{0}$. For a measurable edge $\phi$, define a section $S_{\phi}$, by

$$
S_{\phi}(x)= \begin{cases}0 & \text { if } x \notin \operatorname{Dom}(\phi) \\ \zeta_{\phi(x)} & \text { otherwise }\end{cases}
$$

Let $\psi_{i}$ be a countable family of measurable edges from Lemma 2.2. We make $l^{2}\left(s^{*} x\right)$ into a measurable field by equipping it with the family of sections $S_{\psi_{i}}$.

Let $\phi$ be a measurable edge. We define the corresponding field of operators $\phi_{x}: l^{2}\left(s^{*} x\right) \rightarrow l^{2}\left(s^{*} x\right)$ by

$$
\phi_{x}\left(\zeta_{\alpha}\right)= \begin{cases}\zeta_{\alpha \beta} & \text { if } \exists \beta \in \operatorname{Im}(\phi): r(\alpha)=s(\beta) \\ 0 & \text { otherwise }\end{cases}
$$

Let $f \in L^{\infty}\left(\mathcal{G}_{0}\right)$. We define the corresponding field of operators $f_{x}: l^{2}\left(s^{*} x\right) \rightarrow$ $l^{2}\left(s^{*} x\right)$ by first fixing a measurable function on $\mathcal{G}_{0}$ which is a representative for $f$, we also call it $f$, and by putting

$$
f_{x}\left(\zeta_{\alpha}\right)=f(r(\alpha)) \cdot \zeta_{\alpha}
$$

Given $T \in \mathbb{C} \mathcal{G}$ represented by a finite sum $\sum \phi_{i} \cdot f_{i}$, we define a field of operators $T_{x}: l^{2}\left(s^{*} x\right) \rightarrow l^{2}\left(s^{*} x\right)$ by first fixing representatives for $f_{i}$ and putting $T_{x}=\sum\left(\phi_{i}\right)_{x}\left(f_{i}\right)_{x}$.

2.7. Proposition. There is an isomorphism of Hilbert spaces $L^{2}(\mathcal{G})$ and

$$
\int^{\oplus} l^{2}\left(s^{*} x\right) d \mu(x)
$$

which sends a function $F: \mathcal{G} \rightarrow \mathbb{C}$ to a section

$$
x \mapsto \sum_{\gamma \in s^{*} x} F(\gamma) \cdot \zeta_{\gamma}
$$

Under this isomorphism elements of $\mathbb{C G}$ are decomposable. Operator $T \in \mathbb{C G}$ corresponds to the operator $\int^{\oplus} T_{x} d \mu(x)$. Furthermore,

$$
\tau_{\mathcal{G}}(T)=\int\left\langle T_{x}\left(\zeta_{x}\right), \zeta_{x}\right\rangle_{l^{2}\left(s^{*} x\right)} d \mu(x) .
$$

Proof. The statements about the decomposition of $T$ and the trace follow from the formula (2.3) through a direct computation.

We need to check (1) that the formula (2.3) defines a measurable element of the field $\int^{\oplus} l^{2}\left(s^{*} x\right) d \mu(x)$, (2) that this field is square-summable, and that the resulting map of Hilbert spaces is (3) isometric and (4) surjective.

(1) For each measurable edge $S_{\phi}$ we need to check that the function

$$
x \mapsto\left\langle S_{\phi}(x), \sum_{\gamma \in s^{*} x} F(\gamma) \cdot \zeta_{\gamma}\right\rangle_{l^{2} s^{*} x}
$$


is measurable. By definition of $S_{\phi}$, this function is non-zero only on $\operatorname{Dom}(\phi)$, where it is equal to

$$
\left\langle\zeta_{\phi(x)}, \sum_{\gamma \in s^{*} x} F(\gamma) \cdot \zeta_{\gamma}\right\rangle_{l^{2} s^{*} x}
$$

which is equal to $F(\phi(x))$.

(2) and (3) Due to Lemma 2.2, we have

$$
\langle F, F\rangle_{L^{2} \mathcal{G}}=\int_{\mathcal{G}}|F(\gamma)|^{2} d \mu(\gamma)=\int \sum_{\gamma \in s^{*} x}|F(\gamma)|^{2} d \mu(x)
$$

which is equal to

$$
\int\left\langle\sum_{\gamma \in s^{*} x} F(\gamma) \cdot \zeta_{\gamma}, \sum_{\gamma \in s^{*} x} F(\gamma) \cdot \zeta_{\gamma}\right\rangle_{l^{2} s^{*} x} d \mu(x)
$$

(4) Let an element of $\int^{\oplus} l^{2}\left(s^{*} x\right) d \mu(x)$ be given by a measurable section $F(x) \in$ $l^{2}\left(s^{*} x\right)$. Define $F \in L^{2}\left(\mathcal{G}_{0}\right)$ as $F(\gamma)=\left\langle F(s(\gamma)), \zeta_{\gamma}\right\rangle$. By (2.3), the image of $F$ is the section $F(x)$.

\section{2-D. Relation groupoids with finite orbits}

The measurable structure on the field $x \mapsto l^{2}(\mathcal{G} x)$ of Hilbert spaces is given by the sections

$$
S_{\psi_{i}}= \begin{cases}0 & \text { if } x \notin \operatorname{Dom}(\phi) \\ \zeta_{r\left(\psi_{i}(x)\right)} & \text { otherwise }\end{cases}
$$

where $\psi_{i}$ is the countable family of measurable edges from Lemma 2.2. If $\mathcal{G}$ is a relation groupoid then, under the standard identification of $s^{*} x$ with $\mathcal{G} x$, it is the same measurable structure as the one on the field $x \mapsto l^{2}\left(s^{*} x\right)$.

We say that a groupoid $\mathcal{G}$ has finite orbits, if almost all points in $\mathcal{G}_{0}$ have finite orbits. Note that if $\mathcal{G}$ is a groupoid with finite orbits, then there exists a fundamental domain, i.e. a measurable subset $D \subset \mathcal{G}_{0}$ such that every finite orbit intersects $D$ exactly once. In this case, we define the trace $\tau_{D}$ on decomposable operators on

by the formula

$$
\int_{D}^{\oplus} l^{2}(\mathcal{G} x) d \mu(x)
$$

$$
\tau_{D}\left(\int_{D}^{\oplus} T_{x} d \mu(x)\right)=\int_{D} \operatorname{tr}\left(T_{x}\right) d \mu(x) .
$$

2.8. Proposition. Let $\mathcal{G}$ be a relation groupoid with finite orbits, and let $D$ be a fundamental domain of $\mathcal{G}$. There is a trace-preserving ${ }^{*}$-representation of $\mathbb{C G}$ on $\int_{D}^{\oplus} l^{2}(\mathcal{G} x) d \mu(x)$, which sends an operator $T \in \mathbb{C} \mathcal{G}$ to

$$
\int_{D}^{\oplus} T_{x} d \mu(x)
$$

In particular, for a self-adjoint operator $T$, the spectral measure of $T$ is the same as the spectral measure of $\int_{D}^{\oplus} T_{x} d \mu(x)$.

Proof. Let $D^{c}$ be the complement of $D$. We have the direct sum decomposition

$$
\int^{\oplus} l^{2}(\mathcal{G} x) d \mu(x)=\int_{D}^{\oplus} l^{2}(\mathcal{G} x) d \mu(x) \oplus \int_{D^{c}}^{\oplus} l^{2}(\mathcal{G} x) d \mu(x)
$$


and the corresponding decomposition of the operator $T$ :

$$
\int T_{x} d \mu(x)=\int_{D}^{\oplus} T_{x} d \mu(x) \oplus \int_{D^{c}}^{\oplus} T_{x} d \mu(x) .
$$

It follows that $T \mapsto \int_{D}^{\oplus} T_{x}$ is a ${ }^{*}$-representation. Thus, we need only the equality of the traces.

Using the standard identification of $\mathcal{G} x$ with $s^{*} x$,

$$
\tau_{D}(T)=\int_{D} \sum_{\gamma \in s^{*} x}\left\langle T_{x} \zeta_{\gamma}, \zeta_{\gamma}\right\rangle d \mu(x)
$$

Because of Lemma 2.2,

$$
\tau_{D}(T)=\int_{s^{-1}(D)}\left\langle T_{s(\gamma)} \zeta_{\gamma}, \zeta_{\gamma}\right\rangle d \mu(\gamma)
$$

which, by Lemma 2.1, is equal to

$$
\int_{\mathcal{G}_{0}}\left\langle T_{d(x)} \zeta_{x}, \zeta_{x}\right\rangle d \mu(x)
$$

where $d(x)$ is the unique point in $D \cap \mathcal{G} x$.

In a relation groupoid, for any two points $x, y$ in the same orbit we have $T_{x}=$ $T_{y}: l^{2}(\mathcal{G} x) \rightarrow l^{2}(\mathcal{G} x)$. It is enough to check it for $T=f \in L^{\infty}(X)$ and for $T=\phi$, where $\phi$ is a measurable edge. In both cases it is a straight-forward computation.

Therefore, we have

$$
\tau_{D}(T)=\int_{\mathcal{G}_{0}}\left\langle T_{x} \zeta_{x}, \zeta_{x}\right\rangle d \mu(x)=\tau_{\mathcal{G}}(T)
$$

Equality of the spectral measures follows from Lemma 2.6.

Let $A=\left(\phi_{i}\right)_{i \in I}$ be a family of measurable edges. An $A$-graph is an oriented graph whose edges are labeled by elements of $A$. The $A$-graphing of $\mathcal{G}_{0}$ is the $A$-graph whose vertices are elements of $\mathcal{G}_{0}$; there is an oriented edge from $x$ to $y$ with label $\phi_{i}$ if, for some $\beta \in \operatorname{Im}\left(\phi_{i}\right), s(\beta)=x, r(\beta)=y$. The $A$-graphing of $\mathcal{G} x$ is the full sub- $A$-graph of the $A$-graphing of $\mathcal{G}_{0}$ whose vertices are points of $\mathcal{G} x$.

An $A$-graph in $\mathcal{G}$ is an $A$-graph isomorphic to the $A$-graphing of $\mathcal{G} x$ for some $x \in \mathcal{G}_{0}$. For an $A$-graph $g$ in $\mathcal{G}$, let $[g]$ denote its isomorphism class of $A$-graphs, let

$$
U_{[g]}:=\left\{x \in \mathcal{G}_{0}: A \text {-graphing of } \mathcal{G} x \text { is isomorphic (as an } A \text {-graph) to } g\right\},
$$

and $\mu_{\mathcal{G}}[g]:=\mu\left(U_{[g]}\right)$. When $\mathcal{G}$ is understood, we write $\mu[g]$.

The subring of $\mathbb{C} \mathcal{G}$ generated over $\mathbb{C}$ by elements of $A$ and their inverses is denoted by $\mathbb{C}_{A} \mathcal{G}$. Note that it is ${ }^{*}$-closed.

Given an $A$-graph $g$ and $\phi \in A$ we define $\phi_{g}: l^{2}(g) \rightarrow l^{2}(g)$ by

$$
\left\langle\phi_{g}\left(\zeta_{v}\right), \zeta_{w}\right\rangle= \begin{cases}1 & \text { there is an edge with label } \phi \text { from } v \text { to } w \\ 0 & \text { otherwise }\end{cases}
$$

For $T \in \mathbb{C}_{A} \mathcal{G}$ and an $A$-graph $g$ in $\mathcal{G}$, we define $T_{g}: l^{2} g \rightarrow l^{2} g$ by first fixing a representative $T=\sum c_{i} \phi_{i}$, where $c_{i} \in \mathbb{C}, \phi_{i} \in A \cup A^{-1}$, and putting $T_{g}:=\sum c_{i}\left(\phi_{i}\right)_{g}$. That this is well-defined follows from the observation that $T_{g}$ is conjugate to $T_{x}$ for any $x$ such that the $A$-graphing of $\mathcal{G} x$ is isomorphic to $x$. In fact, every 
isomorphism of $A$-graphs between $g$ and the $A$-graphing of $\mathcal{G} x$ is an intertwining operator.

2.9. Corollary. Let $\mathcal{G}$ be a relation groupoid with finite orbits, $D$ be a fundamental domain, and $g$ be a finite $A$-graph in $\mathcal{G}$ with $\mu[g] \neq 0$. The map $\phi \mapsto \phi_{g}$ extends to $a^{*}$-representation of $\mathbb{C}_{A} \mathcal{G}$ on $l^{2} g$. The image of $T \in \mathbb{C}_{A} \mathcal{G}$ under this representation is $T_{g}$.

Furthermore, consider the Hilbert space

$$
\oplus_{[g]} l^{2} g
$$

where the sum is over different isomorphism classes of $A$-graphs in $\mathcal{G}$ with $\mu[g] \neq 0$. The map $T \mapsto \oplus T_{g}$ is a faithful ${ }^{*}$-representation of $\mathbb{C}_{A} \mathcal{G}$ on the above Hilbert space, and

$$
\tau_{\mathcal{G}}(T)=\sum_{[g]} \frac{\mu[g]}{|V(g)|} \operatorname{tr}\left(T_{g}\right) .
$$

Proof. Let $D$ be a fundamental domain and let $V$ the complement of $U_{[g]}$ in $\mathcal{G}_{0}$. We have a direct sum decomposition

$$
\int_{D}^{\oplus} l^{2}(\mathcal{G} x) d \mu(x)=\int_{U_{[g]} \cap D}^{\oplus} l^{2}(\mathcal{G} x) d \mu(x) \oplus \int_{V \cap D}^{\oplus} l^{2}(\mathcal{G} x) d \mu(x)
$$

and the corresponding direct sum of operators

$$
\int_{D} T_{x} d \mu(x)=\int_{U_{[g]} \cap D}^{\oplus} T_{x} d \mu(x) \oplus \int_{V \cap D}^{\oplus} T_{x} d \mu(x),
$$

which shows that $T \mapsto \int_{U_{[g]} \cap D}^{\oplus} T_{x} d \mu(x)$ is a representation of $\mathbb{C}_{A}$.

To show that $T \rightarrow T_{g}$ is a representation we construct an intertwining operator between $\int_{U_{[g]} \cap D}^{\oplus} l^{2}(\mathcal{G} x)$ and the direct integral $\int_{U_{[g]} \cap D}^{\oplus} l^{2} g$ of the constant field $l^{2} g$. For each $x \in U_{[g]} \cap D$ we need to choose, in a measurable way, an isomorphism between the $A$-graphing of $\mathcal{G} x$ and $g$. Observe that given $x \in \mathcal{G} x$ and $v \in V(g)$, there is at most one such isomorphism which sends $x$ to $v$, since different outgoing vertices of a given vertex have different labels. Let us order the vertices of $g$. The field of intertwiners between $\int_{U_{[g]} \cap D}^{\oplus} l^{2}(\mathcal{G} x)$ and $\int_{U_{[g]} \cap D}^{\oplus} l^{2} g$ is defined as $x \mapsto$ the isomorphism between the $A$-graphing of $\mathcal{G} x$ and $g$ which sends $x$ to the smallest possible $v \in V(g)]$.

To prove that the representation $T \mapsto \oplus T_{g}$ is faithful, it is enough to check that (2.4) holds. We have

$$
\tau_{\mathcal{G}}(T)=\int_{D} \operatorname{tr}\left(T_{x}\right) d \mu(x)=\sum_{[g]} \int_{U_{[g]} \cap D} \operatorname{tr}\left(T_{x}\right) d \mu(x) .
$$

Note that, on the right side, each integrand is a constant function equal to $\operatorname{tr}\left(T_{g}\right)$. The claim follows by noting that $\mu\left(U_{[g]} \cap D\right)=\frac{\mu[g]}{|V(g)|}$.

Given an $A$-graph $g$ and an edge $e$, let $L(e)$ denote the label of $e$. Given a (not-necessarily directed) path $p$ consisting of edges $e_{1}, e_{2}, \ldots, e_{k}$, the label of $p$ is

$$
L(p):=\prod_{i=1}^{n} L\left(e_{i}\right)^{\varepsilon_{i}}
$$

where $\varepsilon_{i}=1$, if $p$ crosses $e_{i}$ respecting the orientation of $e_{i}$, and $\varepsilon_{i}=-1$ otherwise. 
An $A$-graph is uniquely labeled if for every closed path $p$ the measurable edge $L(p)$ is the identity embedding $\mathcal{G}_{0} \rightarrow \mathcal{G}$ restricted to some set.

2.10. Corollary. Let $\mathcal{G}$ be a groupoid and $A$ be a family of measurable edges in $\mathcal{G}$. Suppose that all the orbits in $\mathcal{G}(A)$ are finite and that for almost every object $x$, the A-graphing of $\mathcal{G}(A) x$ is uniquely labeled. Let $T \in \mathbb{C}_{A} \mathcal{G}$. Then $T$ has pure-point spectrum and the von Neumann dimension of the eigenspace for a given $\kappa \in \mathbb{C}$ is equal to

$$
\sum_{[g]} \frac{\mu_{\mathcal{G}(A)}[g]}{|V(g)|} \operatorname{dim} \operatorname{ker}\left(T_{g}-\kappa\right),
$$

where the sum is taken over isomorphism classes of A-graphs in $\mathcal{G}(A)$.

Proof. The assumption that almost all $x$ have uniquely-labeled $A$-graphings implies that $\mathcal{G}(A)$ is a relation groupoid, and $\mathcal{G}(A)$ has finite orbits by assumption. The statement follows by applying the previous corollary to $\mathcal{G}(A)$, since the spaces $l^{2} g$ are finite-dimensional and the von Neumann dimension arising from the standard trace is the standard dimension.

\section{EXAMPLES}

\section{3-A. Action groupoids and Pontryagin duality}

Let $\Gamma$ be a discrete countable group, $(X, \mu)$ be a probability measure space and $\rho: \Gamma \curvearrowright X$ be a right measure preserving action, which is not necessarily free. The action groupoid $\mathcal{G}(\rho)$ is a measured groupoid whose space of objects is $X$, and whose space of morphisms is $X \times \Gamma$. The structure maps are given by $s(x, \gamma)=x$, $r(x, \gamma)=\rho(\gamma)(x)$. Composition of $(x, \alpha)$ and $(\rho(\alpha)(x), \beta)$ is $(x, \alpha \beta)$. The inverse of $(x, \gamma)$ is $\left(\rho(\gamma)(x), \gamma^{-1}\right)$.

Each element $\gamma \in \Gamma$ gives rise to a measurable edge $x \mapsto(x, \gamma)$, denoted also by $\gamma$, whose domain of definition is all of $X$.

For the rest of this subsection, $(X, \mu)$ is a compact abelian group with the normalized Haar measure and the action $\rho: \Gamma \curvearrowright X$ is by continuous group automorphisms. The action $\widehat{\rho}$ of $\Gamma$ on the Pontryagin dual $\widehat{X}$ of $X$ is defined as $\widehat{\rho}(\gamma)(f)(x)=f\left(\rho\left(\gamma^{-1}\right)(x)\right)$, where $f: X \rightarrow \mathbb{C}$ is an element of $\widehat{X}$.

For more information on Pontryagin duality see e.g. [Fol95]. In particular, Pontryagin duality induces a map $P: \widehat{X} \rightarrow L^{\infty}(X)$.

3.1. Proposition. We have a trace-preserving ${ }^{*}$-embedding of the complex group ring $\mathbb{C}\left(\widehat{X} \rtimes_{\widehat{\rho}} \Gamma\right)$ into the groupoid ring $\mathbb{C} \mathcal{G}(\rho)$, which sends $f \in \widehat{X}$ to $P(f)$, and $\gamma \in \Gamma$ to a measurable edge $\gamma$. This embedding will be denoted by $P \otimes 1$.

In particular, if $T=T^{*} \in \mathbb{C}\left(\widehat{X} \rtimes_{\widehat{\rho}} \Gamma\right)$, then $T$ and $P \otimes 1(T)$ have the same spectral measures.

Proof. To begin with, we show that the map $\sum \widehat{c}_{i} \cdot a_{i} \cdot \gamma_{i} \mapsto \sum a_{i} \cdot \gamma_{i}, c_{i} \in \mathbb{C}$, $a_{i} \in \widehat{X}, \gamma_{i} \in \Gamma$, is a ring homomorphism. It is well-defined, since every element of $\mathbb{C}\left(\widehat{X} \rtimes_{\widehat{\rho}} \Gamma\right)$ can be written in a unique way as $\sum \widehat{c}_{i} \cdot a_{i} \cdot \gamma_{i}$. It certainly is a ring homomorphism when restricted to $\mathbb{C} \Gamma$ and to $\mathbb{C} \widehat{X}$. The standard presentation of a semi-direct product and Lemma 2.3(4) imply that it is a homomorphism on all of $\mathbb{C}\left(\widehat{X} \rtimes_{\widehat{\rho}} \Gamma\right)$. 
Lemma 2.3(3) implies that the *-operation is preserved.

By linearity, it is enough to check the trace-preservation on an element of the form $\widehat{a} \cdot \gamma \in \mathbb{C}\left(\widehat{X} \rtimes_{\widehat{\rho}} \Gamma\right)$, where $\widehat{a} \in \widehat{X}, \gamma \in \Gamma$. The group ring trace of $\widehat{a} \cdot \gamma$ is equal to 1 if $\widehat{a}$ and $\gamma$ are the neutral elements of $\widehat{X}$ and $\Gamma$ respectively, and is equal to 0 otherwise.

We consider three cases.

(1) Both $\widehat{a}$ and $\gamma$ are the neutral elements. Then $a$ is the function on $X$ constantly equal to 1 and $\tau_{\mathcal{G}(\rho)}(P \otimes 1(\widehat{a} \cdot \gamma))=\left\langle\chi_{0}, \chi_{0}\right\rangle=1$.

(2) $\gamma$ is not the neutral element. Then the functions $\chi_{0}$ and $\gamma\left(\chi_{0}\right)$ have disjoint supports and, therefore, also $\chi_{0}$ and $(a \gamma)\left(\chi_{0}\right)$ have disjoint supports, so $\left\langle(a \gamma)\left(\chi_{0}\right), \chi_{0}\right\rangle=0$.

(3) $\gamma$ is the neutral element, but $\widehat{a}$ is not. Then, it follows from Pontryagin duality that $a$ is a non-constant function on $\mathcal{G}_{0}$, and the trace we have to compute is equal to $\left\langle a, \chi_{0}\right\rangle=\int a d \mu$. Let $x, y \in \mathcal{G}_{0}$ with $a(x) \neq a(y)$. We use that $a$ is a group homomorphism and invariance of Haar measure to get

$$
\int a(z) d \mu(z)=\int a(x z) d \mu(z)=a(x) \int a(z) d \mu(z) .
$$

Repeating with $y$ in place of $x$ we obtain $a(x) \int a(z) d \mu(z)=a(y) \int a(z) d \mu(z)$, which is possible only if $\int a(z) d \mu(z)=0$.

The statement about the spectral measures follows from Lemma 2.6.

3.2. Lemma. Let $\mathbb{Z}\left(\frac{1}{2}\right)$ be the subring of $\mathbb{Q}$ generated by $\mathbb{Z}$ and $\frac{1}{2}$. If $X$ is a product of infinitely many copies of $\mathbb{Z} / 2 \mathbb{Z}$ indexed by a set $I$, then the image of $\mathbb{Z}\left(\frac{1}{2}\right)\left(\widehat{X} \rtimes_{\widehat{\rho}} \Gamma\right)$ under $P \otimes 1$ is generated over $\mathbb{Z}\left(\frac{1}{2}\right)$ by measurable edges $\gamma \in \Gamma$ and the characteristic functions of cylinder sets.

Proof. Let $R \subset \mathbb{C G}$ be the ring generated by characteristic functions of cylinder sets and measurable edges $\gamma \in \Gamma$.

First, we show that image of $\mathbb{Z}\left(\frac{1}{2}\right)\left(\widehat{X}_{\rtimes_{\widehat{\rho}}} \Gamma\right)$ is contained in $R$. Clearly, $(P \otimes$ $1)(\Gamma) \subset R$. Note that $\widehat{X}$ is a direct sum of infinitely many copies of $\mathbb{Z} / 2 \mathbb{Z}$ indexed by $I$. Let $g_{i}$ be the generator of $\mathbb{Z} / 2 \mathbb{Z}$ corresponding to the index $i \in I$. Direct computation shows that $P \otimes 1\left(\frac{e+g_{i}}{2}\right)$ is the characteristic function of the cylinder set $\left\{\left(x_{j}\right) \in \prod_{I} \mathbb{Z} / 2 \mathbb{Z}: x_{i}=0\right\}$. Also $P \otimes 1(e)$ is a characteristic function of a cylinder set (namely, of the whole $X)$. The statement follows, since $\mathbb{Z}\left(\frac{1}{2}\right)(\widehat{X})$ is generated, as a $\mathbb{Z}\left(\frac{1}{2}\right)$-ring, by $\frac{e+g_{i}}{2}$ and $e$.

In the other direction, we just saw that the characteristic functions of cylinder sets $\left\{\left(x_{j}\right) \in \prod_{I} \mathbb{Z} / 2 \mathbb{Z}: x_{i}=0\right\}$ are in the image. Since the constant function 1 is also in the image, it follows that characteristic function of $\left\{\left(x_{j}\right) \in \prod_{I} \mathbb{Z} / 2 \mathbb{Z}: x_{i}=\right.$ $1\}$ is in the image as well. Every cylinder set is an intersection of sets of those two types, so the claim follows.

\section{3-B. Computation of Grigorchuk \& Żuk}

We now show how Corollary 2.10 is used in practice. We start by computing the von Neumann dimension of the kernel of a random walk operator on the group $\mathbb{Z} / 2 \mathbb{Z} \imath \mathbb{Z}$. This was originally done, by different methods, by R. Grigorchuk and A. Żuk in [GŻ01]. Compare also [DS02]. 
Recall that the lamplighter group $\mathbb{Z} / 2 \mathbb{Z} \imath \mathbb{Z}$ is defined as $\mathbb{Z} / 2 \mathbb{Z} \oplus \mathbb{Z} \rtimes \mathbb{Z}$, where the action of $\mathbb{Z}$ on $\mathbb{Z} / 2 \mathbb{Z} \oplus \mathbb{Z}$ is by the shift i.e. $\left[t\left(\left(x_{i}\right)\right)\right]_{j}=x_{j+1}$,

Let $X=\mathbb{Z} / 2 \mathbb{Z}^{\mathbb{Z}}$. Given $\varepsilon_{-k}, \ldots, \varepsilon_{l} \in \mathbb{Z} / 2 \mathbb{Z}$, the set

$$
\left\{\left(x_{i}\right) \in X: x_{-k}=\varepsilon_{-k}, \ldots, x_{l}=\varepsilon_{l}\right\}
$$

is denoted by

$$
\left[\varepsilon_{-k} \cdots \underline{\varepsilon_{0}} \cdots \varepsilon_{l}\right]
$$

and its characteristic function is denoted by

$$
\chi\left[\varepsilon_{-k} \ldots \underline{\varepsilon_{0}} \ldots \varepsilon_{l}\right] .
$$

Similarly the set $\left\{\left(x_{i}\right) \in X: x_{-1}=0\right\}$ is denoted by [0.] and its characteristic function by $\chi[0 \dot{-}]$. Concrete elements from the set $\left[\varepsilon_{-k} \ldots \underline{\varepsilon_{0}} \ldots \varepsilon_{l}\right]$ are denoted by $\left(\varepsilon_{-k} \ldots \underline{\varepsilon_{0}} \ldots \varepsilon_{l}\right)$.

3.3. Theorem ([GZ̈01]). Let $T$ be the element in the rational group ring of the lamplighter group given by $T=\frac{1}{2}\left(t+t^{-1}+t g+g t^{-1}\right)$, where $t$ is the generator of $\mathbb{Z}$, and $g \in \mathbb{Z} / 2 \mathbb{Z} \oplus \mathbb{Z}$ is a characteristic function $\mathbb{Z} \rightarrow \mathbb{Z} / 2 \mathbb{Z}$ of $\{0\} \subset \mathbb{Z}$. Then $\operatorname{dim}_{v N} \operatorname{ker} T=\frac{1}{3}$.

Proof. Let $X=\{0,1\}^{\mathbb{Z}}$ with the standard product measure, and let $\rho: \mathbb{Z} \curvearrowright X$ be the Bernoulli shift action, i.e. $\left[\rho(t)\left(\left(x_{i}\right)\right)\right]_{j}=x_{j+1}$, where $t$ is the distinguished generator of $\mathbb{Z}$.

Note that $T=t \cdot \frac{1+g}{2}+t^{-1} \cdot \frac{1+t(g)}{2}$, and that the Pontryagin dual of $\frac{1+g}{2}$ is $\chi[\underline{0}]$ and of $\frac{1+t(g)}{2}$ is $\chi\left[0 \cdot \dot{\sim}^{\prime}\right.$. Therefore, by Proposition 3.1, the spectral measure of $T$ is the same as the spectral measure of the operator $t_{[\underline{0}]}+t_{[0-j]}^{-1} \in \mathcal{G}(\rho)$. Let us call the latter operator $T$ as well.

Now, we want to use Corollary 2.10 for the family $A=\left\{t_{[0]}\right\}$ (note that inverse of the measurable edge $t_{[\underline{0}]}$ is the measurable edge $\left.t_{\left[0_{-}\right]}^{-1}\right)$.

Orbits of $\mathcal{G}(A)$ are as follows: the orbit of a point $x_{k}=\left(1 \underline{0} 0^{k-1} 1\right), k>1$, consists of points $\rho\left(t^{i}\right)(x), i=0, \ldots, k$. The $A$-graphing of $\mathcal{G}(A) x_{k}$, denote it by $g_{k}$, has a directed edge from $\rho\left(t^{i-1}\right)(x)$ to $\rho\left(t^{i}\right)(x)$ for $i=1, \ldots k$. It follows that $T_{g}$ is the (directed) random walk operator on the graph

with $k+1$ vertices. Clearly $\mu\left[g_{k}\right]=\frac{k+1}{2^{k+2}}$.

The only other points are of the form $x_{0}=(1 \underline{1})$. Their orbits consist of a single point and the corresponding $A$-graphings have no edges. It follows that, in this case, $T_{g}=0$, and that $\mu[g]=\frac{1}{4}$.

Recall that the kernel of the random walk operator on $k$ vertices is 1-dimensional if $k$ is odd and 0-dimensional otherwise. By Corollary 2.10 we have

$$
\operatorname{dim}_{\mathrm{vN}} \operatorname{ker} T=\sum_{l=0}^{\infty} \frac{1}{2^{2 l+2}}=\frac{1}{3} .
$$

If we put more effort into computing the spectra of the random walk operators on the graphs $g_{k}$ in the proof, we could compute the whole spectral measure of $T$ (see [Ġ̈01] or [DS02]). 


\section{3-C. Percolation theory, theorem of Lehner, Neuhauser \& Woess}

We now present a theorem of F. Lehner, M. Neuhaser and W. Woess from [LNW08]. It is a generalization of the computation from the previous subsection and a special case of Corollary 2.10.

Let $\Gamma$ be a finitely generated group, $A$ be a finite set of generators for $\Gamma$, and $C$ be the associated directed Cayley graph. Vertices of $C$ are elements of $\Gamma$, and there is an edge from $\alpha$ to $\beta$ if and only if $\alpha \gamma=\beta$, for some $\gamma \in A$ (so, if $A$ is a symmetric set of generators, then between each two vertices of $C$ there are either none or two edges). An animal is a full connected subgraph of $C$ which contains the neutral element $e \in \Gamma$. The $A$-boundary of an animal $L$ is the set $\partial_{A} L:=\{\alpha \in V(C)-V(L): \exists \beta \in V(L)$ such that there is an edge from $\alpha$ to $\beta\}$.

Let $\bar{L}$ denote the subgraph of $C$ whose set of vertices is $V(L) \cup \partial_{A} L$, and whose set of edges contains all edges of $L$ and additionally those edges of $C$ which connect vertices of $L$ with points of $\partial_{A} L$.

Given a natural number $p$ and a finite animal $L$, denote

$$
\mu_{p}(\bar{L}):=\left(\frac{1}{p}\right)^{|L|} \cdot\left(\frac{p-1}{p}\right)^{\left|\partial_{A} L\right|} .
$$

Let $T_{\bar{L}}$ denote the (oriented) random walk operator $l^{2} \bar{L} \rightarrow l^{2} \bar{L}$.

Given a point $x \in \mathbb{Z} / p \mathbb{Z}^{\Gamma}$ let $C_{0}(x)$ be the full subgraph of $C$ with $V\left(C_{0}(x)\right)=$ $\{\alpha \in \Gamma: x(\alpha)=0\}$, and let $L(x)$ be the animal whose set of vertices is equal to the connected component of $C_{0}(x)$ containing $e \in \Gamma$ (if $e \notin C_{0} G$ then $L(x)$ is the empty animal).

For $q \in[0,1]$, let $\nu_{q}$ denote the product measure on $\mathbb{Z} / p \mathbb{Z}^{\Gamma}$ of a fixed measure $\nu$ on $\mathbb{Z} / p \mathbb{Z}$ such that $\nu(\{0\})=q, \nu(\{1,2, \ldots, p-1\})=1-q$. We say that a parameter $q$ is subcritical for $\Gamma$ with respect to $A$ if animals $L(x)$ are $\nu_{q}$-almost surely finite. Subcriticality does not depend on $p$ or a choice of $\nu$.

For example, for $\Gamma=\mathbb{Z}$ with the standard generating set, every $q<1$ is subcritical. More generally, for the free group $F_{k}$ with the standard generating set every $q<\frac{1}{2 k-1}$ is subcritical. It is known that there exists $q_{c} \in[0,1]$ such that subcritical parameters are precisely those smaller than $q_{c}$. See [BS96] for more information on percolation theory.

3.4. Theorem ([LNW08]). Let $G=\mathbb{Z} / p \mathbb{Z} 々 \Gamma$, let $\pi \in \mathbb{Q} \mathbb{Z} / p \mathbb{Z}$ be the projection $\pi:=\frac{1}{p} \cdot \sum_{x \in \mathbb{Z} / p \mathbb{Z}} x$, let $T \in \mathbb{Q} G$ be defined as

$$
T:=\sum_{\gamma \in A} \gamma \pi
$$

Suppose that the parameter $\frac{1}{p}$ is subcritical for $\Gamma$ with respect to $A$. Then the spectral measure of $T+T^{*}$ is pure-point and

$$
\operatorname{dim}_{v N} \operatorname{ker}\left(T+T^{*}\right)=\sum_{L} \frac{\mu_{p}(\bar{L})}{|\bar{L}|} \cdot \operatorname{dim} \operatorname{ker}\left(T_{\bar{L}}+T_{\bar{L}}^{*}\right),
$$

where the sum is over all finite animals.

Proof. The proof is analogous to the one in the previous subsection. 
Let $X=\mathbb{Z} / p \mathbb{Z}^{\Gamma}$ with the normalized Haar measure $\mu$ and let the action $\rho: \Gamma \curvearrowright X$ be the Bernoulli shift, i.e.

$$
\left[\rho(\gamma)\left(x_{\alpha}\right)\right]_{\beta}:=x_{\beta \gamma}
$$

The action groupoid $\mathcal{G}(\rho)$ is denoted by $\mathcal{G}$.

Let $[\underline{0}] \subset X$ be the set $\{f \in X: f(e)=0\}$. The image of $T$ under the Pontryagin duality map $P \otimes 1: \mathbb{Q}(\mathbb{Z} / p \mathbb{Z} \imath \Gamma) \rightarrow \mathcal{G}(\rho)$ is the element

$$
\sum_{\gamma \in A} \gamma \cdot \chi_{[0]}
$$

and, by Proposition 3.1, the spectral measure of $T$ is the same as the spectral measure of $(P \otimes 1)(T)$. Let us call the latter operator $T$ as well.

We want to use Corollary 2.10 for the family of measurable edges $\left\{\gamma_{[\underline{0}]}, \gamma \in A\right\}$, which we also call $A$.

Let $x \in X$. The $A$-graphing of the $\mathcal{G}(A)$-orbit of $x$ can be identified (as an oriented graph) with $\overline{L(x)}$, and under this identification $T_{g}$ corresponds to $T_{\overline{L(g)}}$. By the assumption on $p \mu$-almost all animals $L(x)$ are finite and so almost all orbits of $\mathcal{G}(A)$ are finite. Almost all orbits of $\mathcal{G}(A)$ are also uniquely labeled, since the action $\rho$ is essentially free. If we denote the $A$-graphing of the $\mathcal{G}(A)$ orbit of $x$ by $g(x)$ then we have $\mu[g(x)]=\mu_{p}(L(x))$, and so the result follows from Corollary 2.10, since $T+T^{*} \in \mathbb{C}_{A} \mathcal{G}(A)$.

The author was informed by Franz Lehner that is an open problem to determine whether $\frac{1}{p}>q_{c}$ implies that the continuous part appears in the spectral measure of the random walk operator $T+T^{*}$ above, even for groups $\mathbb{Z}^{k}, k>1$. Surprisingly to the author, the conjecture seems to be that for $k=2$ the continuous part does not appear.

The advantage Corollary 2.10 has over the methods of [LNW08] is that it works for many more operators. For example, we can often find an operator $T \in \mathbb{Q}(\mathbb{Z} / p \mathbb{Z}\{\Gamma$ such that the relevant $A$-graphings consist of a prescribed family of graphs $g$, and the operators $T_{g}$ are more arbitrary than the random walk operators. This flexibility is used later in the thesis (Theorem 4.11), and in [Gra10] to show existence of transcendental $l^{2}$-Betti numbers arising from the group $\mathbb{Z} / 2 \mathbb{Z} \imath Z$.

As an easy example, suppose we wanted to obtain an operator $T$ in $\mathbb{Q}(\mathbb{Z} / p \mathbb{Z} \imath \Gamma)$ such that computing the spectral measure of $T$ boils down to computing spectral measures of the random walk operators on animals themselves (and not on $\bar{L}$, as above). Then we would take the preimage under $P \otimes 1: \mathbb{Q}(\mathbb{Z} / p \mathbb{Z} \imath \Gamma) \rightarrow \mathcal{G}(A)$ of the operator $S+S^{*}$, where

$$
S:=\sum_{\gamma \in A} \gamma \cdot \chi([\underline{0} \stackrel{\gamma}{\rightarrow} 0]),
$$

and $[\underline{0} \stackrel{\gamma}{\rightarrow} 0]:=\{x \in X: x(e)=x(\gamma)=0\}$.

\section{TURING DYNAMiCAL SYSTEMS}

\section{4-A. Definitions and basic properties}

Let $(X, \mu)$ be a probability measure space and $\rho: \Gamma \curvearrowright X$ be a right probability measure preserving action of a countable discrete group $\Gamma$ on $X$. 
4.1. Definition. A dynamical hardware is the following data: $(X, \mu)$, the action $\rho$, and a division $X=\bigcup_{i=1}^{n} X_{i}$ of $X$ into disjoint measurable subsets.

For brevity, we denote such a dynamical hardware by $(X)$.

Suppose now that we are given a dynamical hardware $(X)$ and we choose three additional distinguished disjoint subsets of $X$, each of which is a union of certain $X_{i}$ 's: the initial set $I$, the rejecting set $R$, and the accepting set $A$ (all or some of them might be empty). Furthermore, suppose that for every set $X_{i}$, we choose one element $\gamma_{i}$ of the group $\Gamma$ in such a way that the elements corresponding to the sets $X_{i}$ which are subsets of $R \cup A$ are equal to the neutral element of $\Gamma$.

Define a map $T_{X}: X \rightarrow X$ by

$$
T_{X}(x):=\rho\left(\gamma_{i}\right)(x) \quad \text { for } x \in X_{i} \text {. }
$$

4.2. Definition. A dynamical software for a given dynamical hardware $(X)$ is the following data: the distinguished sets $I, A$ and $R$, the choice of elements $\gamma_{i}$, and the map $T_{X}$, subject to the conditions above. The map $T_{X}$ will be called the Turing map, and the whole dynamical software will be denoted by $\left(T_{X}\right)$.

4.3. Definition. A Turing dynamical system is a dynamical hardware $(X)$ together with a dynamical software $\left(T_{X}\right)$ for $(X)$. We will denote such a Turing dynamical system by $\left(X, T_{X}\right)$.

4.4. Proposition. In any Turing system $\left(X, T_{X}\right)$, the Turing map $T_{X}$ is measurecontracting, i.e., for every measurable set $U \subset X$ the set $T_{X}(U)$ is measurable and $\mu\left(T_{X}(U)\right) \leq \mu(U)$. If $T_{X}$ is injective on $U$ then $\mu\left(T_{X}(U)\right)=\mu(U)$.

Proof. $T_{X}(U)$ is measurable since $T_{X}$ is finite-to-one and measurable.

Define $U_{i}:=U \cap X_{i}$. By the definition of $T_{X}$, we have that $T_{X}\left(U_{i}\right)=\rho\left(\gamma_{i}\right)\left(U_{i}\right)$. But all the maps $\rho\left(\gamma_{i}\right)$ are, by Definition 4.1, measure-preserving, and so the claim follows.

4.5. Definition. Let $\left(X, T_{X}\right)$ be a Turing dynamical system.

- A $T_{X}$-chain is a sequence of points $\left(x_{i}\right)$ in $X$, indexed by $\mathbb{Z}$ or $\mathbb{N}$, such that $T_{X}\left(x_{j}\right)=x_{j+1}$. If the $T_{X^{-}}$chain is indexed by $\mathbb{N}$, then we demand that $T_{X}$ maps no point in $X$ to $x_{0}$, and, in this case, we say $x_{0}$ is the starting point of the $T_{X}$-chain $\left(x_{i}\right)$. Similarly, if for some $j$ we have $T_{X}\left(x_{j}\right)=x_{j}$, then we call $x_{j}$ the final point of the $T_{X^{-}}$chain.

- A finite $T_{X}$-chain is one with a finite number of elements belonging to it. If $\left(\alpha_{i}\right)$ is a finite $T$-chain which has the starting point and the final point, then we will denote it also by $\left(\alpha_{0}, \alpha_{1}, \ldots, \alpha_{k}\right)$, understanding that $\alpha_{k}$ is the final point of $\left(\alpha_{i}\right)$ and $\alpha_{k-1}$ is not.

- If the final point of some $T_{X^{-}}$chain lies in a set $U \subset X$ then we say that this $T_{X^{-}}$chain ends in $U$. Similarly, if the starting point of some $T_{X^{-}}$chain lies in $U$ then we say that this $T_{X^{-}}$-chain begins in $U$ or starts in $U$.

- If $p$ is the starting point of some $T_{X^{-}}$chain then we will say that this $T_{X^{-}}$ chain starts at $p$, and similarly for final points.

4.6. Definition. Let $\left(X, T_{X}\right)$ be a Turing dynamical system.

- A fundamental $T_{X}$-chain is a $T_{X^{-}}$-chain which starts in $I$ and ends in $A$. 
- The first fundamental set, or simply the fundamental set of $\left(X, T_{X}\right)$ is the subset $F_{1}$ of $I$ consisting of all starting points of fundamental $T_{X^{-}}$ chains.

- The second fundamental set of $\left(X, T_{X}\right)$ is the subset $F_{2}$ of $A$ consisting of all final points of fundamental $T_{X}$-chains.

4.7. Definition. Both the first and the second fundamental set of $\left(X, T_{X}\right)$ are measurable. Indeed, for example, the first fundamental set is nothing but

$$
\bigcup_{i=1}^{\infty}\left(T_{X}^{-i}(A) \cap I\right)-T_{X}(X)
$$

and $T_{X}(X)$ is measurable by Proposition 4.4. Therefore, we can define the first fundamental value, or simply the fundamental value, of $\left(X, T_{X}\right)$ as the measure of its first fundamental set, and similarly for the second fundamental value. They will be denoted by, respectively, $\Omega_{1}\left(X, T_{X}\right)$ and $\Omega_{2}\left(X, T_{X}\right)$.

In the next definition, the expression almost all $T_{X}$-chains and similar ones should be taken to mean that there exists a family of $T_{X}$-chains such that the set of all points which are elements of $T_{X}$-chains from this family is of full measure.

4.8. Definition. We say that a Turing dynamical system $\left(X, T_{X}\right)$

- is strongly attracting, if almost every $T_{X^{-}}$chain ends in either $R$ or $A$ (in particular almost every $T_{X^{-}}$-chain has a final point.)

- has finite chains, if almost all $T_{X^{-}}$-chains are finite.

- has disjoint fundamental chains, if almost all fundamental $T_{X}$-chains are pairwise disjoint (as sets.)

- does not restart, if $\mu\left(T_{X}(X) \cap I\right)=0$.

4.9. Proposition. If $\left(X, T_{X}\right)$ has disjoint fundamental chains, then

$$
\Omega_{1}\left(X, T_{X}\right)=\Omega_{2}\left(X, T_{X}\right)
$$

Proof. Consider the map $E$ from the first fundamental set $F_{1}$ to the second fundamental set $F_{2}$, given by

$$
E(x):=\text { the final point of the } T_{X^{-}} \text {chain starting at } x
$$

By assumption, this map is injective almost everywhere. On the other hand, we can express $F_{1}$ as a countable disjoint union

$$
F_{1}=\bigcup_{i=1}^{\infty} T_{X}^{-i}\left(F_{2}\right) \cap F_{1}
$$

and it is clear that $E$ restricted to $T_{X}^{-i}\left(F_{2}\right) \cap F_{1}$ is equal to $T_{X}^{i}$. Therefore, the claim follows by applying Proposition 4.4.

4.10. Proposition. If $\left(X, T_{X}\right)$ is strongly attracting then it has finite chains.

Proof. Let $C^{0} \subset X$ denote the set of final points of all $T_{X}$-chains. $C^{0}$ consists precisely of the fixed points of $T_{X}$. Since the system is strongly attracting, the set of fixed points of $T_{X}$ is $A \cup R \cup Z$, where $Z$ is some set of measure 0 .

Similarly define $C^{i}, i>0$, to be the set of points "which are at distance $i$ from a final point". That is, we let

$C^{i}:=\left\{x \in X: \quad T_{X}^{i}(x)\right.$ is a final point, $T^{j}(x)$ is not a final point for $\left.j<i\right\}$. 
Since $C^{i+1}=T_{X}^{-1}\left(C^{i}\right)-\cup_{j \leq i} C^{i}$, we see inductively that the sets $C^{i}$ are measurable. It is clear that these sets are disjoint, and because the system is strongly attracting, we know that

$$
\bigcup_{i \geq 0} C^{i}
$$

is of measure 1 . Therefore, it is enough to see that, for every $i$, the measure of the set

$$
\left\{x \in C^{i}: \quad x \text { is an element of an infinite } T_{X^{-}} \text {-chain }\right\}
$$

is 0 . But for a given $i$ the above set is equal to

$$
\bigcap_{j>i} T_{X}^{j-i}\left(C^{j}\right)
$$

Now, we note that, because $C^{j}$ 's are disjoint, we have

$$
\lim _{j \rightarrow+\infty} \mu\left(C^{j}\right)=0 .
$$

This, together with the fact that $T_{X}$ is measure-contracting (by Proposition 4.4), proves the claim.

\section{4-B. Expressing the fundamental values as von Neumann dimensions}

Let us now fix a Turing dynamical system $\left(X, T_{X}\right)$. Define $T$ to be an element of the groupoid ring $\mathbb{C G}(\rho)$ given by $T:=\sum_{i=1}^{n} \gamma_{i} \chi_{i}$, where $\chi_{i}$ 's are characteristic functions of the respective $X_{i}$ 's.

Given two operators $A$ and $B$ on some Hilbert space, note that ker $A^{*} A+B^{*} B=$ ker $A \cap \operatorname{ker} B$.

Let $S \in \mathbb{C} \mathcal{G}(\rho)$ be defined as

$$
S:=\left(T+\chi_{X}-\chi_{I}-\chi_{A}-\chi_{R}\right)^{*}\left(T+\chi_{X}-\chi_{I}-\chi_{A}-\chi_{R}\right)+\chi_{A} .
$$

4.11. Theorem. Let $\left(X, T_{X}\right)$ be a Turing dynamical system which does not restart and is strongly attracting. Then the von Neumann dimension of kernel of $S$ is equal to $\mu(I)-\Omega_{2}\left(X, T_{X}\right)$.

Proof. Let $A$ be the family of measurable edges $\gamma_{i \mid X_{i}}$. Note that $S \in \mathbb{C}_{A} \mathcal{G}(\rho)$.

We want to use the formula from Corollary 2.10, i.e.

$$
\sum_{[g]} \frac{\mu_{\mathcal{G}(A)([g])}}{|V(g)|} \cdot \operatorname{dim} \operatorname{ker} S_{g}
$$

where the sum is over distinct isomorphism classes of $A$-graphs. In order to be able to do so, we need to check the assumptions of Corollary 2.10 hold.

Lemma. (1) In the groupoid $\mathcal{G}(A)$, the orbit $\mathcal{G}(A) x$ of almost any point $x$ is finite.

(2) For almost any point $x$ the A-graphing of $\mathcal{G}(A) x$ is uniquely labeled.

Proof. Note that in the $A$-graphing of $\mathcal{G}(A) x$ there is an oriented edge between two points $y$ and $z$ precisely when $T_{X}(y)=z$. Because our system is strongly attracting, $A$-graphings of $\mathcal{G}(A)$-orbits are oriented rooted trees with a self-loop at the root (see Figure 1), whose label is the neutral element of $\Gamma$ (restricted to either $A$ or $R$ ). This implies that $T$-graphings of orbits are uniquely labeled.

Let $\mathcal{G}(A)_{0}^{\infty}$ be the set of points $x$ in $\mathcal{G}(A)_{0}=\mathcal{G}_{0}=X$ such that $\mathcal{G}(A) x$ is infinite, and let $E=\mathcal{G}(A)_{0}^{\infty} \cap(A \cup R)$. Therefore $E$ is precisely the set of final points of 


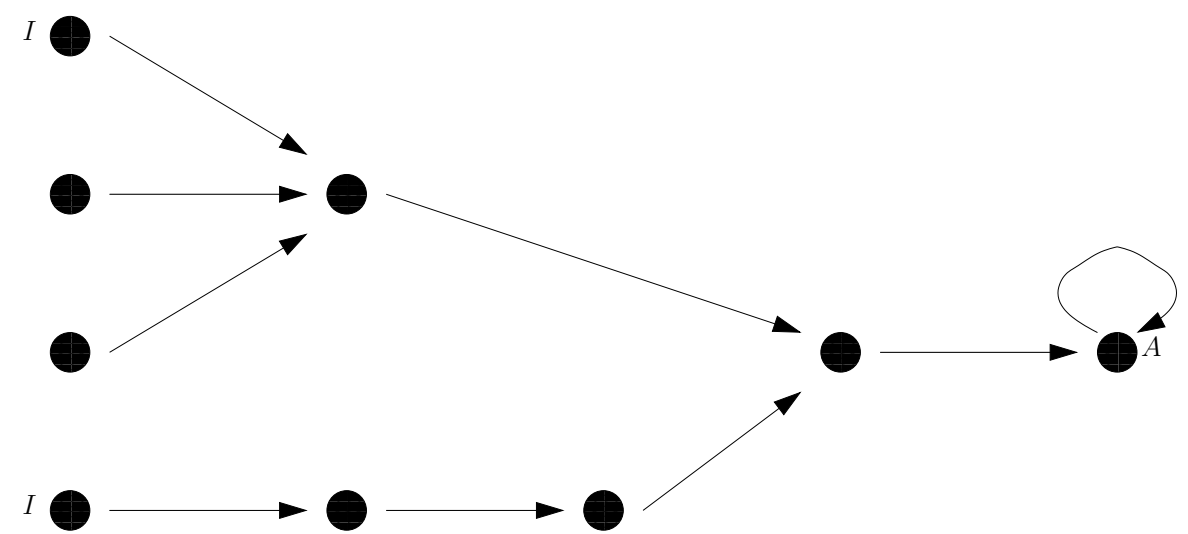

Figure 1. An example $A$-graphing of a $\mathcal{G}(A)$-orbit. Some of the starting points belong to $I$, and the final point belongs to either $A$ or $R$. The only loop in the graph is the self-loop at the final point whose label is the neutral element of $\Gamma$.

infinite $T_{X}$-chains, and as such it has, by Proposition 4.10, measure 0. But every point whose connected component is infinite is contained in one of countably many translates of $E$ by elements of $\Gamma$, and so the first statement also follows.

Let us fix $x \in X$ and let $g$ be the $A$-graphing of $\mathcal{G}(A) x$. Thanks to the formula (4.1) it is enough to show that $\operatorname{dim} \operatorname{ker} S_{g}$ is equal to

$$
|V(g) \cap I|-|V(g) \cap A| .
$$

Let us first compute the kernel of $T_{g}+\left(\chi_{X}\right)_{g}-\left(\chi_{I}\right)_{g}-\left(\chi_{A}\right)_{g}-\left(\chi_{R}\right)_{g}$. Note that $T_{g}$ is defined by the formula

$$
\left\langle T_{g}\left(\zeta_{v}\right), \zeta_{w}\right\rangle= \begin{cases}1 & \text { there is an edge with from } v \text { to } w \\ 0 & \text { otherwise }\end{cases}
$$

The operator $\left(\chi_{X}\right)_{g}-\left(\chi_{I}\right)_{g}-\left(\chi_{A}\right)_{g}-\left(\chi_{R}\right)_{g}$ acts identically on vectors $\zeta_{v}$ for $v \notin A \cup I \cup R$ and is null on other vectors $\zeta_{v}$.

Let $x_{1}$ be any starting vertex of $g$ which belongs to the initial set $I$, and let $\left(x_{1}, x_{2}, \ldots x_{k}\right)$ be a $T_{X}$-chain. Note that the vector

$$
\xi\left(x_{1}\right):=\zeta_{x_{1}}-\zeta_{x_{2}}+\ldots \pm \zeta_{x_{k}}
$$

is in the kernel of $T_{g}+\left(\chi_{X}\right)_{g}-\left(\chi_{I}\right)_{g}-\left(\chi_{A}\right)_{g}-\left(\chi_{R}\right)_{g}$. Furthermore if $x, y, \ldots, z$ are different starting vertices then the vectors $\xi(x), \xi(y), \ldots, \xi(z)$ are linearly independent.

Lemma. The linear span of the set $\{\xi(x): x \in V(g) \cap I\}$ is equal to the kernel of $T_{g}+\left(\chi_{X}\right)_{g}-\left(\chi_{I}\right)_{g}-\left(\chi_{A}\right)_{g}-\left(\chi_{R}\right)_{g}$

Proof. We just saw that the linear span of $\{\xi(x): x \in V(g) \cap I\}$ is in the kernel, so it is enough to see that it generates the whole kernel. If this was not the case than there would be a vector $\eta$ in the kernel such that $\left\langle\eta, \zeta_{x}\right\rangle=0$ for every vertex $x \in I$. Let $y$ be a maximal (with respect to the relation generated by oriented edges) vertex of $g$ such that $\left\langle\eta, \zeta_{y}\right\rangle \neq 0$. 
Write $\eta=\zeta_{y}+\eta^{\prime}$, where $\eta^{\prime}$ is a linear combination of vectors $\zeta_{z}$ for $y>z$. It follows that $\left\langle T_{g}+\left(\chi_{X}\right)_{g}-\left(\chi_{I}\right)_{g}-\left(\chi_{A}\right)_{g}-\left(\chi_{R}\right)_{g} \eta^{\prime}, \zeta_{y}\right\rangle=0$, and so

$$
\begin{aligned}
0=\left\langle T_{g}+\left(\chi_{X}\right)_{g}-\left(\chi_{I}\right)_{g}-\left(\chi_{A}\right)_{g}\right. & \left.-\left(\chi_{R}\right)_{g} \eta, \zeta_{y}\right\rangle= \\
& =\left\langle T_{g}+\left(\chi_{X}\right)_{g}-\left(\chi_{I}\right)_{g}-\left(\chi_{A}\right)_{g}-\left(\chi_{R}\right) \zeta_{y}, \zeta_{y}\right\rangle .
\end{aligned}
$$

But if $y$ is not a final vertex then $\left\langle T_{g} \zeta_{y}, \zeta_{y}\right\rangle=0$, and $\left\langle\left(\chi_{X}\right)_{g}-\left(\chi_{I}\right)_{g}-\left(\chi_{A}\right)_{g}-\right.$ $\left.\left(\chi_{R}\right) \zeta_{y}, \zeta_{y}\right\rangle=1$ (the last equality follows, since by assumption also $\left.y \notin I\right)$, which is a contradiction. And if $y$ is a final vertex then $\left\langle T_{g} \zeta_{y}, \zeta_{y}\right\rangle=1$, and $\left\langle\left(\chi_{X}\right)_{g}-\right.$ $\left.\left(\chi_{I}\right)_{g}-\left(\chi_{A}\right)_{g}-\left(\chi_{R}\right) \zeta_{y}, \zeta_{y}\right\rangle=0$, which is also a contradiction.

Now we need to consider two cases: either the final point of $g$ is in $R$ or $A$. If the first possibility holds then $\chi_{A_{g}}=0$ and $\operatorname{ker} S_{g}=\operatorname{ker} T_{g}+\left(\chi_{X}\right)_{g}-\left(\chi_{I}\right)_{g}-$ $\left(\chi_{A}\right)_{g}-\left(\chi_{R}\right)_{g}$, and then the formula 4.2 holds because the dimension of linear span of $\{\xi(x): x \in I\}$ is precisely $|I|$. If the final point of $g$ is in $A$ then kernel of $\chi_{A}^{g}$ is of codimension 1, and we see that it non-trivially intersects span of $\{\xi(x): x \in I\}$, which implies that kernel of $S^{g}$ has dimension equal to $|I|-1$, which shows that the formula (4.2) holds.

4.12. Corollary. Let $\left(X, T_{X}\right)$ be a Turing dynamical system which does not restart, is strongly attracting, and has disjoint fundamental chains. Then

$$
\operatorname{dim}_{v N} \operatorname{ker} S=\mu(I)-\Omega_{1}\left(X, T_{X}\right) .
$$

Proof. Follows directly from the previous theorem and Proposition 4.9.

\section{TURING DYNAMiCAL SYSTEMS - EXAMPLES}

We want to use presented examples later together with Theorem 4.11 and Proposition 3.1, so we have to assure that the actions are by continuous group automorphisms, which is the reason for an extra degree of complicacy.

\section{5-A. Turing dynamical system associated to a set of natural numbers}

Definition of $X$ and $\Gamma$. $\quad$ Let $X$ be a measure space $M^{\mathbb{Z}} \times S$, where $M:=$ $\mathbb{Z} / 2 \mathbb{Z} \oplus \mathbb{Z} / 2 \mathbb{Z} \oplus \mathbb{Z} / 2 \mathbb{Z}$ should be interpreted as the set of symbols, and $S:=$ $\mathbb{Z} / 2 \mathbb{Z} \oplus \mathbb{Z} / 2 \mathbb{Z} \oplus \mathbb{Z} / 2 \mathbb{Z}$ as the set of states of a Turing machine.

Let $\operatorname{Aut}(M)$ be the group of group automorphisms of $M$; similarly for $\operatorname{Aut}(S)$. Recall that $\operatorname{Aut}(M) \geq \mathbb{Z}$ is defined as $\operatorname{Aut}(M)^{\oplus \mathbb{Z}} \rtimes \mathbb{Z}$, and put

$$
\Gamma=[(\operatorname{Aut}(M) \imath \mathbb{Z}) * \mathbb{Z} / 2 \mathbb{Z}] \times \operatorname{Aut}(S),
$$

where $*$ denotes the free product.

Notation for elements of $X$. Given $m_{-k}, \ldots, m_{0}, \ldots, m_{l} \in M$ and $\sigma \in S$ the set

$$
\left\{\left(\left(n_{i}\right), \tau\right) \in M^{\mathbb{Z}} \times S: n_{-k}=m_{-k}, \ldots, n_{l}=m_{l}, \tau=\sigma\right\}
$$

is denoted by

$$
\left[m_{k} m_{k-1} \ldots, m_{-1} \underline{m_{0}} m_{1} \ldots m_{l}\right][\sigma] .
$$

Given $\sigma \in S$, the set

$$
\bigcup_{m \operatorname{im} M}[\underline{m}][\sigma]
$$


is denote by []$[\sigma]$, and given $m \in M$ the set

$$
\bigcup_{\sigma \in S}[\underline{m}][\sigma]
$$

is denoted by $[\underline{m}][]$. by

A concrete element from the set $\left[m_{k} m_{k-1} \ldots, m_{-1} m_{0} m_{1} \ldots m_{l}\right][\sigma]$ is denoted

and similarly for ()$[\sigma]$ and $(\underline{m})[]$.

$$
\left(m_{k} m_{k-1} \ldots, m_{-1} \underline{m_{0}} m_{1} \ldots m_{l}\right)[\sigma]
$$

Definition of the action. Let us fix a set of positive natural numbers $\Sigma$. The action $\rho$ of $\Gamma$ on $X$ will depend on $\Sigma$. Whenever we want to stress this dependence we use the symbol $\rho_{\Sigma}$.

Let $\beta^{\prime}$ be the automorphism of $M$ defined by $\beta^{\prime}((1,0,0))=(1,0,0), \beta^{\prime}((0,1,0)=$ $(0,0,1), \beta^{\prime}(0,0,1)=(0,1,0)$. Let $M^{\beta}$ be the set of fixed points of $\beta^{\prime}$. Note that it consists of 4 elements. Let $\beta$ be the automorphism of $M^{\mathbb{Z}}$ defined by

$$
\left(\beta\left(\left(x_{i}\right)\right)\right)_{j}:= \begin{cases}\beta^{\prime}\left(x_{0}\right) & \text { if } j=0 \\ x_{j} & \text { otherwise }\end{cases}
$$

The automorphism $\beta$ will be referred to as normal flip.

Let $B=B_{\Sigma}$ be the following automorphism of $M^{\mathbb{Z}}$ :

$$
\left(B\left(\left(x_{i}\right)\right)\right)_{j}:= \begin{cases}x_{j} & \text { if } j \notin \Sigma \\ \beta^{\prime}\left(x_{j}\right) & \text { otherwise }\end{cases}
$$

The automorphism $B$ will be referred to as oracle flip.

We proceed to describe $\rho$.

The subgroup $\operatorname{Aut}(M) \gtrless \mathbb{Z}<\Gamma$ acts in the standard way on the $M^{\mathbb{Z}}$ coordinate of $X$ : $\operatorname{Aut}(M)$ acts on the 0 -coordinate of $M^{\mathbb{Z}}$ in the natural way, and the generator $t$ of $\mathbb{Z}$ acts by

$$
\left(\rho(t)\left(\left(m_{i}\right)\right)\right)_{j}:=m_{j+1} .
$$

The maps $\rho(t)$ and $\rho\left(t^{-1}\right)$ will be called respectively shift forward, and shift backward.

The subgroup $\operatorname{Aut}(S)<\Gamma$ acts on the $S$ coordinate of $X$ in the natural way.

The generator of $\mathbb{Z} / 2 \mathbb{Z}$ acts by the oracle flip $B$ on $M^{\mathbb{Z}}$; the generator will also be denoted by $B$.

Division of $X$ into disjoint subsets. We choose the following division:

$$
X=\bigsqcup_{m \in M, \sigma \in S}[\underline{m}][\sigma] .
$$

We just finished defining a dynamical hardware $(X)$. When we need to stress its dependence on $\Sigma$, we denote it by $\left(X^{\Sigma}\right)$.

Elements of $S=\mathbb{Z} / 2 \mathbb{Z} \oplus \mathbb{Z} / 2 \mathbb{Z} \oplus \mathbb{Z} / 2 \mathbb{Z}$ will be referred to as [Start], [Search forward for $(0,1,0)]$, [Search backward for $(0,1,0)]$, [Search forward for either $(0,1,0)$ or $(0,0,1)]$, [Dummy state 1], [Dummy state 2], [Dummy state 3], [Dummy state 4]. We do not specify which names correspond to which elements of $S$ - the only important thing is that this assignment is made in such a way that $\operatorname{Aut}(S)$ acts transitively on the first four elements.

We proceed to define a dynamical software for $\left(X^{\Sigma}\right)$. 
Choice of elements of $\Gamma$ for the sets $[\underline{m}][\sigma]$. This is done in Figure 2: arrow between two states $\sigma$ and $\tau$ with a label, for example,

$(0,1,0)$ : normal flip, shift backward

means that the element of $\Gamma$ corresponding to $[(0,1,0)][\sigma]$ is $a \cdot \beta \cdot t^{-1}$, where $a \in \operatorname{Aut}(S)$ is any group automorphism sending $\sigma$ to $\tau$. Since the action is a right action it means in particular that in this example flipping is done before shifting. Similarly, an arrow with label

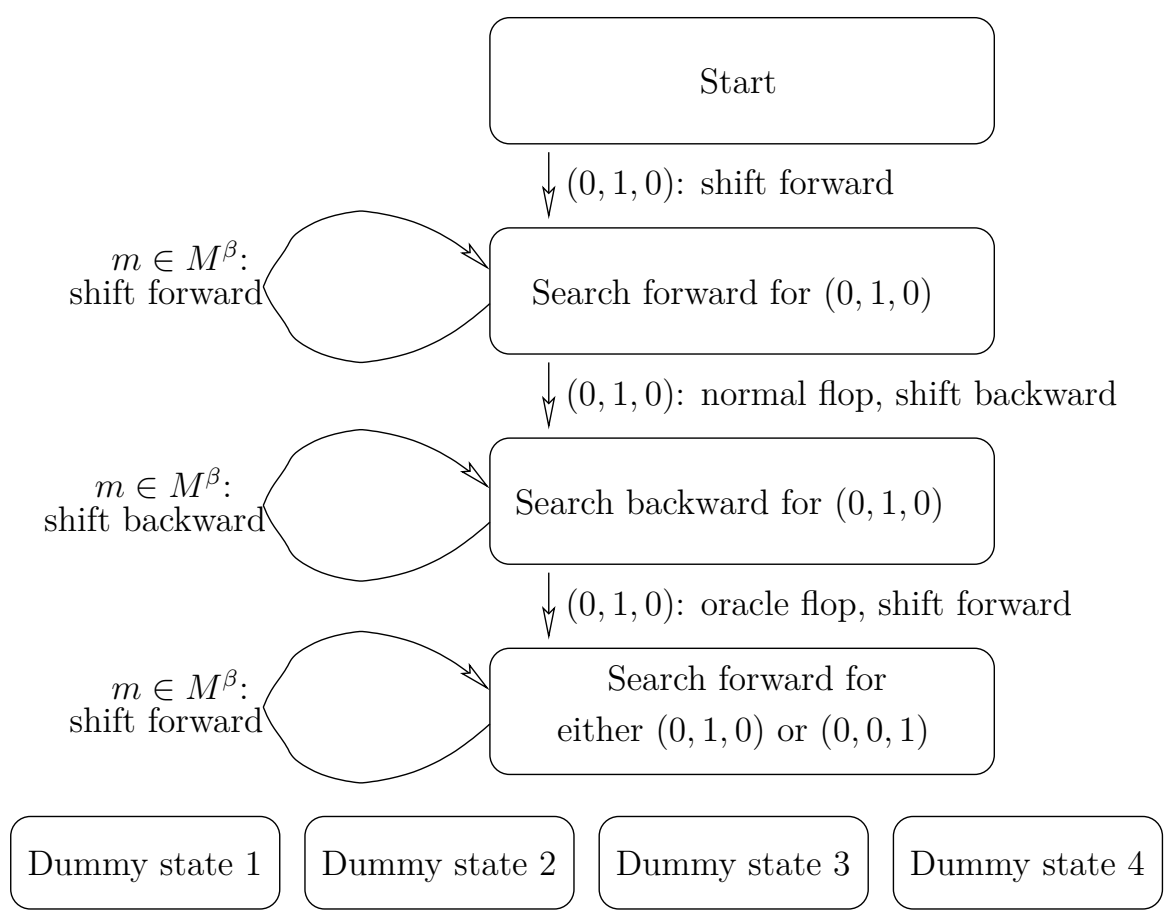

Figure 2. Turing dynamical system $\left(X^{\Sigma}, T_{X}^{\Sigma}\right)$

$m \in M^{\beta}:$ shift forward

joining a state $\sigma$ with itself means that the element of $\Gamma$ corresponding to $[\underline{m}][\sigma]$ for $m \in M^{\beta}$ is $t$.

Finally, if for some state $\sigma$ there is no label with a given symbol $m \in M$ then it means that the element of $\Gamma$ corresponding to $[\underline{m}][\sigma]$ is the neutral element.

Choice of the sets $I, A$ and $R$. . We specify them as follows:

$$
\begin{aligned}
I & :=[(0,1,0)][\text { Start }], \\
A & :=\underline{(0,1,0)]}[\text { Search forward for }(0,1,0) \text { or }(0,0,1)] .
\end{aligned}
$$

As to the set $R$, it is defined as the union of all the sets $[\underline{m}][\sigma]$ whose associated group element is the neutral element, apart from $A$.

Defined software will be denoted by $\left(T_{X}\right)$ or $\left(T_{X}^{\Sigma}\right)$.

\section{5-B. Properties of the system $\left(X, T_{X}\right)$}

5.1. Proposition. The first fundamental set of $\left(X, T_{X}\right)$ is equal to the union

$$
\bigcup_{k \in \Sigma} F_{k} \cup Z
$$


where $F_{k}$ is equal to

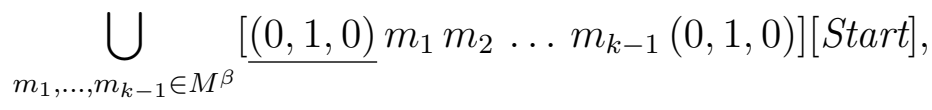

and $Z$ is some set of measure 0 .

Proof. This proposition follows from chasing through Figure 2. First, we show that $F_{k}$ is in the fundamental set for $k \in \Sigma$.

Let $x=\left((0,1,0) m_{1} m_{2} \ldots m_{k-1}(0,1,0)\right)[$ Start $], m_{i} \in M^{\beta}, k \in \Sigma$. Because of the arrow between the first and the second level of Figure 1, we have

$$
T(x)=\left((0,1,0) \underline{m_{1}} m_{2} \ldots m_{k-1}(0,1,0)\right)[\text { Search forward for }(0,1,0)] .
$$

Then, because of the arrow " $m \in M^{\beta}$ : shift forward" on the second level of Figure 2 , we get

$$
T^{k}(x)=\left((0,1,0) m_{1} m_{2} \ldots m_{k-1} \underline{(0,1,0)}\right)[\text { Search forward for }(0,1,0)] .
$$

Because of the arrow between the second and the third level, we see

$$
T^{k+1}(x)=\left((0,1,0) m_{1} m_{2} \ldots \underline{m_{k-1}}(0,0,1)\right)[\text { Search backward for }(0,1,0)] \text {. }
$$

Because of the arrow " $m \in M^{\beta}$ : shift backward" on the third level, we conclude

$$
T^{2 k}(x)=\left(\underline{(0,1,0)} m_{1} m_{2} \ldots m_{k-1}(0,0,1)\right)[\text { Search backward for }(0,1,0)] .
$$

Because of the arrow between the third and the fourth level, and since $k \in \Sigma$, we get that $T^{2 k+1}(x)$ is equal to

$\left((0,1,0) \underline{m_{1}} m_{2} \ldots m_{k-1}(0,0,1)\right)[$ Search forward for either $(0,1,0)$ or $(0,0,1)]$.

Finally, because of the arrow " $m \in M^{\beta}$ : shift forward" on the fourth level, we see that $T^{3 k}(x)$ equals

$$
\left.\left((0,1,0) m_{1} m_{2} \ldots m_{k-1} \underline{(0,0,1)}\right) \text { [Search forward for either }(0,1,0) \text { or }(0,0,1)\right] \text {, }
$$

which is an element of the accepting set.

In the other direction, let

$$
x=\left(\underline{m_{0}} m_{1} \ldots m_{k-1} m_{k}\right)[\sigma]
$$

be an element of the fundamental set of $\left(X, T_{X}\right)$. We can assume $m_{1}, \ldots, m_{k-1} \in$ $M^{\beta}$, and $m_{k} \notin M^{\beta}$, since points $\left(\left(x_{i}\right), \sigma\right) \in X$ such that $x_{i} \in M^{\beta}$ for $i>0$ form a set of measure 0 . We have to prove that (1) $\sigma=[$ Start $],(2) m_{0}=(0,1,0),(3)$ $m_{k}=(0,1,0)$, and $(4) k \in \Sigma$.

(1) and (2) follow from $I=(0,1,0)[$ Start $]$. As before we have

$$
T_{X}^{k}(x)=\left(m_{0} m_{1} \ldots m_{k-1} \underline{m_{k}}\right)[\text { Search forward for }(0,1,0)],
$$

and therefore from the fact that $T_{X}^{k}(x) \notin R$ we get (3). Again, as before we see $T_{X}^{2 k}(x)$ is equal to

$$
\left(\underline{m_{0}} m_{1} \ldots m_{k-1}(0,0,1)\right)[\text { Search backward for }(0,1,0)] \text {. }
$$

Now, suppose that (4) does not hold, i.e. $k \notin \Sigma$. Assume that (4) does not hold. Then because of the arrow between the third and the fourth level, and by the definition of the oracle flip we get that $T_{X}^{2 k+1}(x)$ is

$$
\left(m_{0} \underline{m_{1}} \ldots m_{k-1}(0,0,1)\right) s[\text { Search forward for either }(0,1,0) \text { or }(0,0,1)] \text {, }
$$


which implies that $T_{X}^{3 k}(x)$ is

$\left(m_{0} m_{1} \ldots m_{k-1} \underline{(0,0,1)}\right)[$ Search forward for either $(0,1,0)$ or $(0,0,1)]$,

which is an element of $R$, which contradicts the assumption that $x$ is in the fundamental set.

5.2. Corollary. The first fundamental value of $\left(X, T_{X}\right)$ is equal to

$$
\frac{2}{8^{3}} \sum_{i \in \Sigma} \frac{1}{2^{i}}
$$

Proof. The sets $F_{k}$ are disjoint, and the measure of $F_{k}$ is equal to $\frac{1}{8}\left(\frac{1}{2}\right)^{k-1} \frac{1}{8} \frac{1}{8}=\frac{2}{8^{3}} \frac{1}{2^{k}}$.

5.3. Proposition. The Turing dynamical system $\left(X, T_{X}\right)$ has the following properties.

(i) It doesn't restart.

(ii) It has disjoint fundamental chains.

(iii) It is strongly attracting.

Proof. (i) We have to show that $T_{X}(I) \cap I=\emptyset$. Recall $I=[(0,1,0)][$ Start $]$; from Figure 2 we see that (1) points from outside of [][Start] are not mapped into [][Start], and in particular are not mapped into $I$; (2) points from $I$ are mapped outside of [][Start] and in particular outside of $I$; and (3) Points from [][Start] which are not in $I$ are mapped identically to themselves, and so are also not mapped to I.

(ii) We need to check that there exists a subset of the fundamental set which have the same measure as the fundamental set and such that the mapping

$$
x \mapsto \text { final point of the } T_{X} \text {-chain containing } x
$$

is injective. But in the proof of Proposition 5.1 we saw that if $x \in F_{k}, k \in \Sigma$, and we write $x=\left((0,1,0) m_{1} m_{2} \ldots m_{k-1}(0,1,0)\right)[$ Start $]$ then the final point of the $T_{X}$-chain containing $x$ is $T^{3 k}(x)$ equal to

$$
\left((0,1,0) m_{1} m_{2} \ldots m_{k-1} \underline{(0,0,1)}\right)[\text { Search forward for either }(0,1,0) \text { or }(0,0,1)] \text {, }
$$

in particular $T^{3 k}(x)$ allows as to recover $x$. In particular the above map is injective on the set $\bigcup_{k \in \Sigma} F_{k}$

For (iii), note the following claim.

Claim. Suppose $x=\left(\left(m_{i}\right), \sigma\right) \in M^{\mathbb{Z}} \times S=X$ is such that its $T_{X}$-chain doesn't end in $A \cup R$. Then there exists $N \in \mathbb{Z}$ such that $m_{j} \in M^{\beta}$ for all $j \geq N$ or for all $j \leq N$.

Proof. The only elements of $\Gamma$ which are applied to the $M^{\mathbb{Z}}$-coordinate are shifts, normal flip and oracle flip. Since they preserve the property "exists $N$ such that $m_{j} \in M^{\beta}$ for all $j \geq N$ or all $j \leq N$ ", it is enough to show that some power $T_{X}^{j}(x)$ has this property. But this is clear from Figure 1: first, there exists a state $\tau$ and $K$ such that $T_{X}^{k}(x) \in[][\tau]$ for $k \geq K$. But the only way it is possible is if either (1) for some $k, T_{X}^{k}(x)$ is in a set $[m][\sigma]$ for which the corresponding element of $\gamma$ is the neutral element, or (2) if we write $T^{K}(x)=\left(\left(n_{i}\right), \tau\right)$ then exists $N$ such that 
$n_{j} \in M^{\beta}$ for all $j>N$ or all $j<N$. The second case is what we want to show, and the first case is not possible because it implies that $T^{k}(x) \in A \cup R$.

Now, (iii) follows, since the set of points $\left(m_{i}\right) \in M^{\mathbb{Z}}$ such that there exists $N$ such that $m_{j} \in M^{\beta}$ for $j>N$ is of measure 0 .

\section{5-C. A "read only" system with irrational fundamental values}

Definition of $Y$ and $\Delta$. Let $Y$ be the measure space $\left(\mathbb{Z} / 2 \mathbb{Z}^{\mathbb{Z}} \times \mathbb{Z} / 2 \mathbb{Z}^{\mathbb{Z}} \times\right.$ $\left.\mathbb{Z} / 2 \mathbb{Z}^{\mathbb{Z}}\right) \times S$, where $S=\mathbb{Z} / 2 \mathbb{Z} \times \mathbb{Z} / 2 \mathbb{Z} \times \mathbb{Z} / 2 \mathbb{Z}$ should again be interpreted as the set of states. However, in this example $\mathbb{Z} / 2 \mathbb{Z}$ should be interpreted as the set of symbols, and $\mathbb{Z} / 2 \mathbb{Z}^{\mathbb{Z}} \times \mathbb{Z} / 2 \mathbb{Z}^{\mathbb{Z}} \times \mathbb{Z} / 2 \mathbb{Z}^{\mathbb{Z}}$ should be interpreted as the set of triples of tapes.

Let $\Delta$ be the group $\mathbb{Z}^{3} \times \operatorname{Aut}(S)$, and let standard generators of $\mathbb{Z}^{3}$ be denoted by $t_{1}, t_{2}$, and $t_{3}$. They will be also referred to as, respectively, shift forward tape 1, shift forward tape 2 , and shift forward tape 3. Similarly $t_{i}^{-1}$ will be referred to as shift backward tape $i$.

Definition of the action. The action $\rho: \Delta \curvearrowright Y$ is defined as follows. Let $V: \mathbb{Z} / 2 \mathbb{Z}^{\mathbb{Z}} \rightarrow \mathbb{Z} / 2 \mathbb{Z}^{\mathbb{Z}}$ be the shift automorphism, i.e. $V\left(\left(x_{i}\right)\right)_{j}=x_{j+1}$. Define

$$
\begin{aligned}
& \rho\left(t_{1}\right)\left(\left(x_{i}\right),\left(y_{i}\right),\left(z_{i}\right)\right):=\left(V\left(\left(x_{i}\right)\right),\left(y_{i}\right),\left(z_{i}\right)\right) \\
& \rho\left(t_{2}\right)\left(\left(x_{i}\right),\left(y_{i}\right),\left(z_{i}\right)\right):=\left(\left(x_{i}\right), V\left(\left(y_{i}\right)\right),\left(z_{i}\right)\right) \\
& \rho\left(t_{3}\right)\left(\left(x_{i}\right),\left(y_{i}\right),\left(z_{i}\right)\right):=\left(\left(x_{i}\right),\left(y_{i}\right), V\left(\left(z_{i}\right)\right)\right) .
\end{aligned}
$$

$\operatorname{Aut}(S)$ acts in the natural way on the $S$-coordinate.

Notation. Notation is similar to that in Subsection A. Given $a_{-k_{a}}, \ldots, a_{l_{a}}$, $b_{-k_{b}}, \ldots, b_{l_{b}}, c_{-k_{c}}, \ldots c_{l_{c}} \in \mathbb{Z} / 2 \mathbb{Z}$ and $\sigma \in S$, the set

$$
\begin{aligned}
\left\{\left(\left(x_{i}\right),\left(y_{i}\right),\left(z_{i}\right), \tau\right) \in\left(\mathbb{Z} / 2 \mathbb{Z}^{\mathbb{Z}} \times \mathbb{Z} / 2 \mathbb{Z}^{\mathbb{Z}} \times \mathbb{Z} / 2 \mathbb{Z}^{\mathbb{Z}}\right) \times S:\right. & \\
x_{-k_{a}} & \left.=a_{-k_{a}}, \ldots, z_{l_{c}}=c_{l_{c}}, \tau=\sigma\right\}
\end{aligned}
$$

is denoted by

$$
\left[\begin{array}{ccccc}
a_{-k_{a}} & \ldots & \frac{a_{0}}{b_{0}} & \ldots & a_{l_{a}} \\
b_{-k_{b}} & \ldots & \underline{b_{0}} & \ldots & b_{l_{b}} \\
c_{-k_{c}} & \ldots & \underline{c_{0}} & \ldots & c_{l_{c}}
\end{array}\right][\sigma] .
$$

Given $v=\left(v_{1}, v_{2}, v_{3}\right) \in \mathbb{Z} / 2 \mathbb{Z}^{3}$ and $\sigma \in S$, the set

$$
\left[\frac{\underline{v_{1}}}{\underline{v_{2}}}\right][\sigma] \text {. }
$$

is also denoted by $[\underline{v}][\sigma]$ and $\left[\left(v_{1}, v_{2}, v_{3}\right)\right][\sigma]$.

Within the context of above notation, given a natural number $k$ and $\varepsilon \in \mathbb{Z} / 2 \mathbb{Z}$ we denote by $\varepsilon^{k}$ the sequence of $k$ consecutive $\varepsilon$ 's.

Division of $Y$. We choose the following decomposition of $Y$ :

$$
Y=\bigsqcup_{v \in \mathbb{Z} / 2 \mathbb{Z}^{3}, \sigma \in S}[\underline{v}][\sigma] .
$$

We have just defined a dynamical hardware $(Y)$ 
The states - i.e. elements of $S$ - will be referred to as [Start], [Check if the number of 0 's on tape 1 and on tape 2 is the same], [Search backward for 1 on tape 1], [Search forward for 1 on tape 1 and move forward tape 3], [Dummy state 1], [Dummy state 2], [Dummy state 3], [Dummy state 4]. Again we do not specify which of these names correspond to which elements of $S$, but we demand that $\operatorname{Aut}(S)$ acts transitively on the first four of the above states.

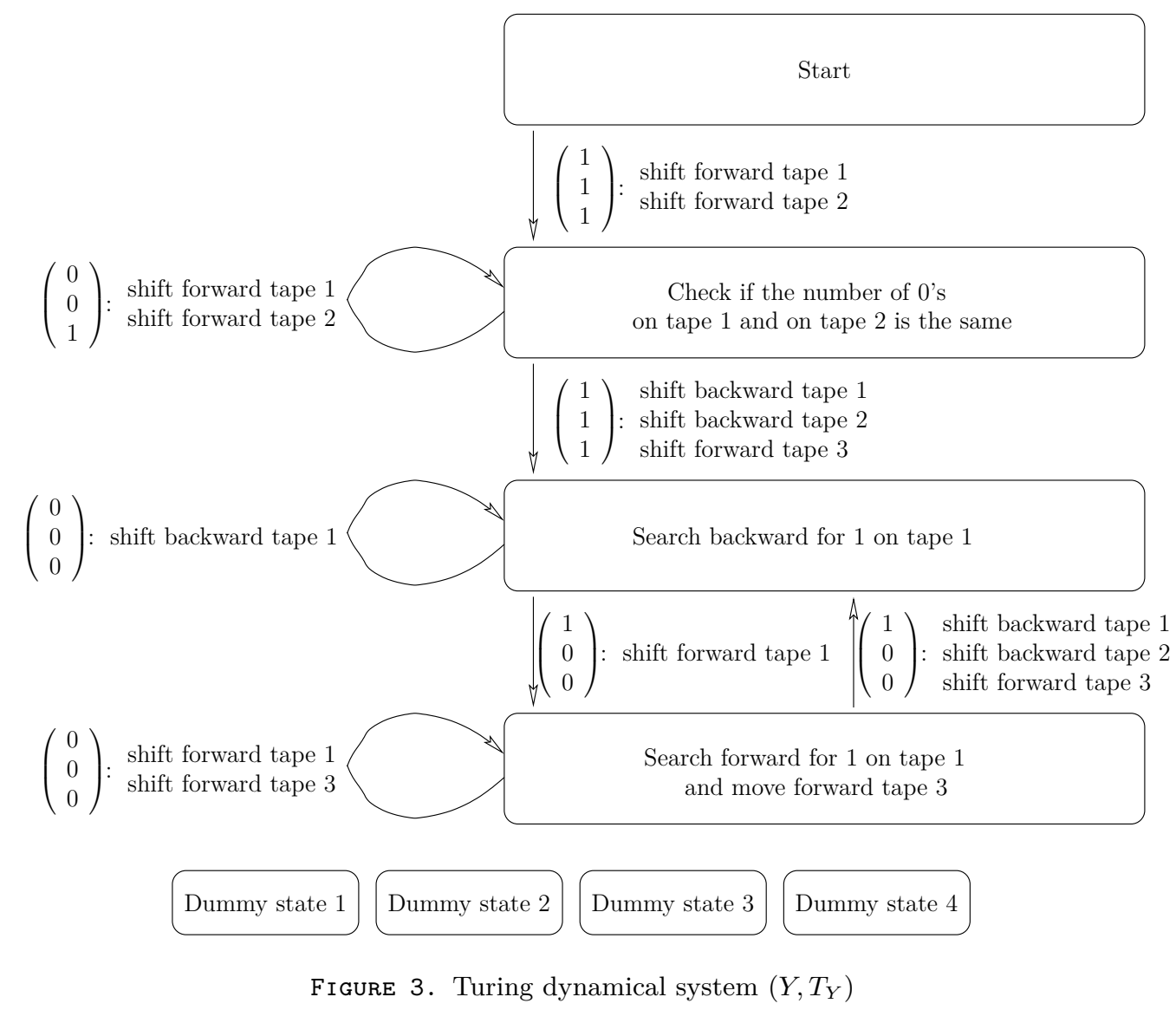

We proceed to define a dynamical software $\left(T_{Y}\right)$ for $(Y)$. This is done using Figure 2, with the same convention as in Subsection A, e.g. an arrow with a label

$$
\left(\begin{array}{l}
1 \\
1 \\
1
\end{array}\right): \begin{aligned}
& \text { shift forward tape } 1 \\
& \text { shift forward tape } 2
\end{aligned}
$$

between states $\sigma$ and $\tau$ means that the element of $\Delta$ associated to the set $[\underline{(1,1,1)}][\sigma]$ is $a \cdot t_{1} \cdot t_{2}$, where $a \in \operatorname{Aut}(S)$ is any group automorphism of $S$ which sends $\sigma$ to $\tau$.

Choice of the sets $A, I$ and $R$. We define them as follows:

$$
\begin{aligned}
I & :=[\underline{(1,1,1)}][\text { Start }], \\
A & :=[\underline{(0,1,1)}][\text { Search backward for } 1 \text { on tape } 1],
\end{aligned}
$$

and the rejecting set $R$ is defined to be the union of all the sets $[\underline{v}][\sigma]$ whose associated group element is the neutral element, apart from $A$. 


\section{5-D. Properties of the system $\left(Y, T_{Y}\right)$}

5.4. Proposition. The first fundamental set of $\left(Y, T_{Y}\right)$ is equal to the union

$$
\bigcup_{k=1}^{\infty} F_{k} \cup Z
$$

where $Z$ is some set of measure 0 and $F_{k}$ is defined to be

$$
\left[\begin{array}{l}
\underline{1} 0^{k} 1 \\
\underline{1} 0^{k} 1 \\
\underline{1} 0^{k^{2}+2 k} 1
\end{array}\right] \text { [Start]. }
$$

Proof. The proof is by chasing Figure 3, fully analogous to the proof of Proposition 5.1 .

5.5. Corollary. The first fundamental value of $\left(Y, T_{Y}\right)$ is equal to

$$
\frac{1}{8} \sum_{k=1}^{\infty} \frac{1}{2^{k^{2}+4 k+6}}
$$

Proof. Indeed, measure of the set $F_{k}$ is equal to $\frac{1}{8} \cdot \frac{1}{2^{k^{2}+2 k}} \cdot \frac{1}{2^{k}} \cdot \frac{1}{2^{k}} \cdot \frac{1}{2^{6}}$ (i.e. " $\frac{1}{8}$ is for states, $\frac{1}{2^{k^{2}+2 k}}$ and $\frac{1}{2^{k}}$ are for 0's, and $\frac{1}{2^{6}}$ is for 1 's'.)

5.6. Proposition. $\left(Y, T_{Y}\right)$ has the following properties.

(i) It does not restart.

(ii) It has disjoint fundamental chains.

(iii) It is strongly attracting.

Proof. (i) and (ii) are proved just like (i) and (ii) of Proposition 5.3.

As for (iii), let $x \in Y$ be a point whose $T_{Y}$-chain doesn't end in $A \cup R$. If $T_{Y^{-}}$chain of $y$ stays on the first two levels of Figure 3 then it means there are infinitely many consecutive 0's to the right on the first two tapes of $y$. Since the set of points which have infinitely many consecutive 0's on any tape is of measure 0 , we can assume that $T_{Y}$-chain of $y$ from some point on stays on the third and the fourth level of Figure 3 (since a $T_{Y}$-chain can not go from the third or fourth level to the first or second level).

Consider two possibilities: (1) $T_{Y}$-chain of $y$ stays from some point on on the third level, or (2) $T_{Y}$-chain of $y$ has infinitely many points on the fourth level.

If (1) holds then because of the arrow

$$
\left(\begin{array}{l}
0 \\
0 \\
0
\end{array}\right) \text { : shift backward tape } 1
$$

we see that the first tape of $y$ has infinitely many consecutive 0's to the left.

If (2) holds then note that the only element of $\Delta$ which acts on the third tape of the $T_{Y}$-chain of $y$ is shift forward. If $T_{Y}$-chain of $y$ is infinite then from Figure 3 we see that shift forward on the third tape is applied infinitely many times. We see also that it can be applied only if there is 0 on the third tape. Therefore in this case there are infinitely many consecutive 0 's on the third tape of $y$. 


\section{Atiyah PROBLEM}

\section{6-A. Preliminaries}

Recall that if $G$ is a (discrete countable) group then there is a trace $\tau_{G}$ (called group trace) on the group ring $\mathbb{C} G$ which extends to a faithful normal trace with respect to the (right) action of $M_{k}(\mathbb{C} G)$ on $\left(l^{2} G\right)^{k}$ for any $k$. Given $T \in M_{k}(\mathbb{C} G)$, von Neumann dimension of the kernel of $T$ is defined as the spectral measure of $T$ with respect to this action and the group trace of the set $\{0\}$. It is denoted by $\operatorname{dim}_{\mathrm{vN}} \operatorname{ker} T$.

Recall that a real number $r$ is an $l^{2}$-Betti number arising from $G$ if for some $k$ there exists $T \in M_{k}(\mathbb{Q} G)$ such that

$$
\operatorname{dim}_{v N} \operatorname{ker} \theta=r .
$$

The set of $l^{2}$-Betti numbers arising from $G$ is denoted by $\mathcal{C}(G)$. Note that if $r \in \mathcal{C}(G)$ then we can always assume that $r=\operatorname{dim}_{\mathrm{vN}} \operatorname{ker} T$ for a positive selfadjoint $T$ of norm $<1$, since $\operatorname{ker} T=\operatorname{ker} T^{*} T=\operatorname{ker} \frac{1}{N} T^{*} T$ for every positive number $N$.

Recall that a set $\Sigma$ of natural numbers is called computable if there exists a Turing machine which lists elements of $\Sigma$ in the increasing order. Equivalently (see e.g. [LS01]), $\Sigma$ is computable if there exists a Turing machine which lists elements of $\Sigma$ in some order (possibly with repetitions), and there exists a Turing machine which lists elements of the complement of $\Sigma$ in some order (possibly with repetitions).

We say that a real number $r$ has a computable binary expansion if the fractional part of $r$ is of the form

for some computable set $\Sigma$.

$$
\sum_{i \in \Sigma} \frac{1}{2^{i}}
$$

In this section we prove Theorems 1.1, 1.3, 1.4, and Corollary 1.2:

Theorem. The set of $l^{2}$-Betti numbers arising from the group $(\mathbb{Z} / 2 \mathbb{Z} \geq \mathbb{Z})^{3}$ contains

$$
\frac{1}{64}-\frac{1}{8} \sum_{k=1}^{\infty} \frac{1}{2^{k^{2}+4 k+6}}
$$

which is a transcendental number.

Theorem. The set of $l^{2}$-Betti numbers arising from finitely generated groups is equal to the set of non-negative real numbers.

Theorem. The set of $l^{2}$-Betti numbers arising from finitely presented groups contains all numbers with computable binary expansions.

Corollary. Let $G$ be a group given by the presentation

$$
\left\langle a, t, s \mid a^{2}=1,[t, s]=1,\left[t^{-1} a t, a\right]=1, s^{-1} a s=a t^{-1} a t\right\rangle .
$$

The set of $l^{2}$-Betti numbers arising from $G^{3}$ contains

$$
\frac{1}{64}-\frac{1}{8} \sum_{k=1}^{\infty} \frac{1}{2^{k^{2}+4 k+6}}
$$


Corollary follows from the fact proven in [GLSŻ00], namely $\mathbb{Z} / 2 \mathbb{Z} \imath \mathbb{Z}$ is a subgroup of $G$, and the following lemma.

6.1. Lemma. Let $H$ be a subgroup of a group $G$. Then $\mathcal{C}(H) \subset \mathcal{C}(G)$.

Proof. The map $M_{k}(\mathbb{C} H) \hookrightarrow M_{k}(\mathbb{C} H)$, induced by the inclusion $H \hookrightarrow G$, is a tracepreserving *-homomorphism. The claim follows from Lemma 2.6.

6.2. Lemma. If $G$ is a discrete countable group and $H$ is a finite group then $|H| \cdot \mathcal{C}(G \times H)=\mathcal{C}(G)$.

Proof. Let $\pi \in \mathbb{Q}(G \times H)$ be the sum

$$
\frac{1}{|H|} \sum_{h \in H} h
$$

Clearly $\pi$ is a projection of trace $\frac{1}{|H|}$ which commutes with $\mathbb{Q} G$. Similarly the $k \times k$ matrix $\pi_{k}$ which has $\pi$ everywhere on the diagonal and 0's elsewhere is of trace $\frac{1}{|H|}$ and commutes with $M_{k}(\mathbb{Q} G)$. We claim that for a positive self-adjoint $T \in M_{k}(\mathbb{Q} G)$ of norm at most 1 we have

$$
\frac{1}{|H|} \cdot \operatorname{dim}_{\mathrm{vN}} \operatorname{ker} T=\operatorname{dim}_{\mathrm{vN}} \operatorname{ker}\left(1-\pi_{k}+\pi_{k} T\right)
$$

Indeed, by functional calculus the right side is equal to the limit of

$$
\tau_{G \times H}\left(\left(1-\left(1-\pi_{k}+\pi_{k} T\right)\right)^{n}\right),
$$

for $n$ going to $+\infty$. The above expression is equal to $\tau_{G \times H}\left(\pi_{k}(1+T)^{n}\right)=\tau_{H}(\pi)$. $\tau\left((1+T)^{n}\right)$. By functional calculus again limit of the latter expressions is equal to $\frac{1}{|H|} \cdot \operatorname{dim}_{\mathrm{vN}} \operatorname{ker} T$. This shows the inclusion $C(G) \subset|H| \cdot \mathcal{C}(G \times H)$

For the other inclusion, note that the regular representation of $H$ induces a ${ }^{*}$-embedding $\mathbb{Q} H \hookrightarrow M_{|H|}(\mathbb{Q})$ such that for $T \in \mathbb{Q} H$ we have $|H| \tau_{H}(T)=\operatorname{tr}(T)$. This induces a ${ }^{*}$-embedding

$$
\iota: \mathbb{Q}(G \times H) \cong \mathbb{Q}(G) \otimes \mathbb{Q}(H) \hookrightarrow \mathbb{Q}(G) \otimes M_{|H|}(\mathbb{Q}) \cong M_{|H|}(\mathbb{Q}(G))
$$

such that for $T \in \mathbb{Q}(G \times H)$ we have $|H| \cdot \tau_{G \times H}(T)=\tau_{G}(\iota(T))$. The result follows from Lemma 2.6.

6.3. Lemma. Let $G$ be a countable discrete group. The set $\mathcal{C} G$ is closed under addition. Furthermore, if $H$ is another countable discrete group, $a \in \mathcal{C}(G), b \in$ $\mathcal{C}(H)$ then $a+b \in \mathcal{C}(G \times H)$.

Proof. The first claim follows from the fact that if $S \in M_{k}(\mathbb{Q} G)$ and $T \in M_{l}(\mathbb{Q} G)$ then $S \oplus T \in M_{k+l}(\mathbb{Q} G)$ has the property

$$
\operatorname{dim}_{\mathrm{vN}} \operatorname{ker}(S \oplus T)=\operatorname{dim}_{\mathrm{vN}} \operatorname{ker} S+\operatorname{dim}_{\mathrm{vN}} \operatorname{ker} T .
$$

The second claims follows from taking $S \in M_{k}(\mathbb{Q} G)$ and $T \in M_{l}(\mathbb{Q} H)$ and observing that for $S \oplus T \in M_{k+l}(\mathbb{Q}(G \times H)$ we also have

$$
\operatorname{dim}_{\mathrm{vN}} \operatorname{ker}(S \oplus T)=\operatorname{dim}_{\mathrm{vN}} \operatorname{ker} S+\operatorname{dim}_{\mathrm{vN}} \operatorname{ker} T \text {. }
$$


6.4. Lemma. Let $\left(X, T_{X}\right)$ be a Turing dynamical system in which $X$ is a compact abelian group $\Pi \mathbb{Z} / 2 \mathbb{Z}$, the action of $\Gamma$ on $X$ is by continuous group automorphisms and the distinguished disjoint subsets $X_{i}$ of $X$ are cylinder sets.

Suppose furthermore that $\left(X, T_{X}\right)$ doesn't restart, has disjoint fundamental chains and is strongly attracting. Then

$$
\mu(I)-\Omega_{1}\left(X, T_{X}\right),
$$

is an $l^{2}$-Betti number arising from the group $\widehat{X} \rtimes_{\widehat{\rho}} \Gamma$.

Proof. By Corollary 2.10, $\mu(I)-\Omega_{1}\left(X, T_{X}\right)$ is equal to $\operatorname{dim}_{\mathrm{vN}} \operatorname{ker} S$, where $S \in \mathcal{G}(\rho)$ is expressed by a finite sum of elements $\gamma_{i} \chi_{i}$, where $\gamma_{i} \in \Gamma$, and $\chi_{i}$ are products of characteristic functions of the sets $X_{i}$. By Lemma 3.2, $S$ is in the image of the Pontryagin map $P \otimes 1: \mathbb{Q}\left(\widehat{X} \rtimes_{\widehat{\rho}} \Gamma\right) \rightarrow \mathcal{G}(\rho)$. Let $\widehat{S}$ be the preimage of $S$. By Proposition 3.1 we get

$$
\operatorname{dim}_{\mathrm{vN}} \operatorname{ker} \widehat{S}=\operatorname{dim}_{\mathrm{vN}} \operatorname{ker} S=\mu(I)-\Omega_{1}\left(X, T_{X}\right) .
$$

\section{6-B. The lamplighter group}

6.5. Theorem. The set of $l^{2}$-Betti numbers arising from the group $(\mathbb{Z} / 2 \mathbb{Z} \backslash \mathbb{Z})^{3}$ contains

$$
\frac{1}{64}-\frac{1}{8} \sum_{k=1}^{\infty} \frac{1}{2^{k^{2}+4 k+6}},
$$

Proof. Note that Turing dynamical system $\left(Y, T_{Y}\right)$ from Subsection 5.C fulfills conditions of Lemma 6.4. We conclude, by Corollary 5.5 that the number

$$
\frac{1}{64}-\frac{1}{8} \sum_{k=1}^{\infty} \frac{1}{2^{k^{2}+4 k+6}}
$$

is an $l^{2}$-Betti number arising from the group $\widehat{Y} \rtimes_{\widehat{\rho}} \Delta$, or more explicitly from the group

$$
\left(\mathbb{Z} / 2 \mathbb{Z}^{\oplus \mathbb{Z}} \oplus \mathbb{Z} / 2 \mathbb{Z}^{\oplus \mathbb{Z}} \oplus \mathbb{Z} / 2 \mathbb{Z}^{\oplus \mathbb{Z}} \oplus \widehat{S}\right) \rtimes_{\widehat{\rho}}(\mathbb{Z} \oplus \mathbb{Z} \oplus \mathbb{Z} \oplus \operatorname{Aut}(S)) .
$$

Note that the copies of $\mathbb{Z}$ act only on respective copies of $\mathbb{Z} / 2 \mathbb{Z} \oplus \mathbb{Z}$, and that they act by the shift. It follows that the above group is isomorphic to the group

$$
(\mathbb{Z} / 2 \mathbb{Z} \imath \mathbb{Z})^{3} \times(\widehat{S} \rtimes \operatorname{Aut}(S)),
$$

so the result follows from Lemma 6.2.

\section{6-C. Finitely generated groups}

Theorem. The set of $l^{2}$-Betti numbers arising from finitely generated groups is equal to the set of non-negative real numbers.

Proof. Note that Turing dynamical system $\left(X^{\Sigma}, T_{X}^{\Sigma}\right)$ from Subsection 5.A fulfills conditions of Lemma 6.4. We conclude, by Corollary 5.2 that the number

$$
\frac{1}{64}-\frac{2}{8^{3}} \sum_{i \in \Sigma} \frac{1}{2^{i}}
$$


is an $l^{2}$-Betti number arising from the group $\widehat{X} \rtimes_{\rho^{\Sigma}} \Gamma$, or more explicitly from the group

$$
\left(\widehat{M}^{\oplus \mathbb{Z}} \times \widehat{S}\right) \rtimes_{\widehat{\rho}}[(\operatorname{Aut}(M) \imath \mathbb{Z}) * \mathbb{Z} / 2 \mathbb{Z}] \times \operatorname{Aut}(S),
$$

so, by Lemma 6.2, also from the group

$$
\widehat{M}^{\oplus \mathbb{Z}} \rtimes_{\widehat{\rho}}((\operatorname{Aut}(M) \imath \mathbb{Z}) * \mathbb{Z} / 2 \mathbb{Z}),
$$

which is easily seen to be finitely generated. This and the fact that $\Sigma$ was arbitrary implies that every number between $\frac{1}{64}-\frac{2}{8^{3}}$ and $\frac{1}{64}$ is an $l^{2}$-Betti number arising from a finitely generated group.

By additivity (Lemma 6.3), we see that there exists a natural number $N=\frac{8^{3}}{64 \cdot 2}$ such that every number between $N-1$ and $N$ with is an $l^{2}$-Betti number arising from a finitely generated group. By additivity again it follows that every number bigger than $N$ is an $l^{2}$-Betti number arising from a finitely generated group.

Let $r$ be an arbitrary positive real number. By the previous paragraph there exists a natural number $k$ such that $k \cdot r$ is an $l^{2}$-Betti number arising from a finitely presented group. The result follows from Lemma 6.2.

\section{6-D. Finitely presented groups}

Theorem. The set of $l^{2}$-Betti numbers arising from finitely presented groups contains all numbers with computable binary expansions.

Proof. Recall that a presentation $\left\langle g_{1}, g_{2}, \ldots \mid r_{1}, r_{2}, \ldots\right\rangle$ is a recursive presentation if there exists an algorithm which lists all the elements of the set $\left\{r_{1}, r_{2}, \ldots\right\}$ in some order (perhaps with repetitions).

Theorem (Higman's Embedding Theorem). If a group has a recursive presentation then it can be embedded into a finitely presented group.

For a proof, see [Hig61]. Higman treated the case of infinitely many generators, although many standard books (e.g. [LS01]) state Higman's theorem only for presentations with finitely many generators.

Lemma. Let $\Sigma$ be a set of natural numbers. And let $\left(X^{\Sigma}, T_{X}^{\Sigma}\right)$ be the Turing dynamical system from Subsection 5-A If $\Sigma$ is computable (i.e. all elements of $\Sigma$ can be listed by a Turing machine and all elements of the complement of $\Sigma$ can be listed by a Turing machine) then the group

$$
\widehat{M}^{\oplus \mathbb{Z}} \rtimes \widehat{\rho^{\Sigma}}((\operatorname{Aut}(M) \imath \mathbb{Z}) * \mathbb{Z} / 2 \mathbb{Z})
$$

has a recursive presentation.

Proof. Recall that when a group $G=\left\langle g_{1}, g_{2}, \ldots \mid p_{1}, p_{2}, \ldots\right\rangle$ acts on a group $H=$ $\left\langle h_{1}, h_{2}, \ldots \mid r_{1}, r_{2}, \ldots\right\rangle$ through an action $\alpha$ then the standard presentation of the semi-direct product $H \rtimes_{\alpha} G$ is

$$
\left\langle h_{2}, h_{2}, \ldots ; g_{1}, g_{2}, \ldots \mid p_{1}, p_{2}, \ldots ; r_{1}, r_{2}, \ldots ; g_{i} h_{j} g_{i}^{-1}=\alpha\left(g_{i}\right)\left(h_{j}\right), i, j=1,2, \ldots\right\rangle \text {. }
$$

When we proceed to write this presentation in the case at hand, the only part which could possibly make it non-algorithmic is the action of $\mathbb{Z} / 2 \mathbb{Z}$. For $m \in$ $\widehat{\mathbb{Z} / 2 \mathbb{Z}^{3}}$ and $j \in \mathbb{Z}$, let $m_{j}$ denote the element $(\ldots, 0, m, 0, \ldots) \in(\widehat{\mathbb{Z} / 2 \mathbb{Z}})^{\oplus} \oplus \mathbb{Z}$, with $m$ on the $j$ 'th place. Let $s$ be the generator of $\mathbb{Z} / 2 \mathbb{Z}$. Recall that Let $\beta^{\prime}$ is 
the automorphism of $M$ defined by $\beta^{\prime}((1,0,0))=(1,0,0), \beta^{\prime}((0,1,0)=(0,0,1)$, $\beta^{\prime}(0,0,1)=(0,1,0)$. and that the action of $s$ on $m_{j}$ is trivial if $j \notin \Sigma$ and is by the Pontryagin dual $\widehat{\beta^{\prime}}$ if $j \in \Sigma$. The relations which we have to write down are of the form:

$$
\begin{array}{ll}
s \cdot m_{j} \cdot s^{-1}=m_{j}, & \text { for } j \notin \Sigma \\
s \cdot m_{j} \cdot s^{-1}=\left[\widehat{\beta^{\prime}}(m)\right]_{j} & \text { for } j \in \Sigma
\end{array}
$$

It is clear that if $\Sigma$ is computable, i.e. there exists an algorithm which lists all elements of $\Sigma$, and there is an algorithm which lists all elements of the complement of $\Sigma$, then there exists also an algorithm which lists all of the above relations.

We saw in the previous subsection that the number

$$
\frac{1}{64}-\frac{2}{8^{3}} \sum_{i \in \Sigma} \frac{1}{2^{i}}
$$

is an $l^{2}$-Betti arising from

$$
\widehat{M}^{\oplus \mathbb{Z}} \rtimes \widehat{\rho^{\Sigma}}((\operatorname{Aut}(M) \imath \mathbb{Z}) * \mathbb{Z} / 2 \mathbb{Z}) .
$$

By Higman's Embedding Theorem and the previous lemma we see that when $\Sigma$ is computable, then (6.2) is an $l^{2}$-Betti number arising from a finitely presented group.

By additivity, we see that there exists a natural number $N=\frac{8^{3}}{64 \cdot 2}$ such that every number between $N-1$ and $N$ with a computable binary expansion is an $l^{2}$-Betti number arising from a finitely presented group. By additivity again, it follows that every number bigger than $N$ with a computable binary expansion is an $l^{2}$-Betti number arising from a finitely presented group.

Let $r$ be an arbitrary positive number with a computable binary expansion. By the previous paragraph there exists a natural number $k$ such that $2^{k} \cdot r$ is an $l^{2}$-Betti number arising from a finitely presented group (since $2^{k} \cdot r$ has also computable binary expansion). The result follows from Lemma 6.2.

\section{REFERENCES}

[Ati76] Michael F. Atiyah. Elliptic operators, discrete groups and von Neumann algebras. In Colloque "Analyse et Topologie" en l'Honneur de Henri Cartan (Orsay, 1974), pages 43-72. Astérisque, No. 32-33. Soc. Math. France, Paris, 1976.

[Aus09] Tim Austin. Rational group ring elements with kernels having irrational dimension, 2009. Preprint, available at http://arxiv.org/abs/0909.2360.

[BS96] Itai Benjamini and Oded Schramm. Percolation beyond $\mathbf{Z}^{d}$, many questions and a few answers. Electron. Comm. Probab., 1:no. 8, 71-82 (electronic), 1996.

[CC10] Cristian S. Calude and Gregory J. Chaitin. What is... a Halting Probability? Not. Am. Math. Soc., 57:236-237, 2010.

[CG86] Jeff Cheeger and Mikhael Gromov. $L_{2}$-cohomology and group cohomology. Topology, 25(2):189-215, 1986.

$\left[\mathrm{DLM}^{+} 03\right]$ Józef Dodziuk, Peter Linnell, Varghese Mathai, Thomas Schick, and Stuart Yates. Approximating $L^{2}$-invariants and the Atiyah conjecture. Comm. Pure Appl. Math., 56(7):839-873, 2003. Dedicated to the memory of Jürgen K. Moser.

[Dod77] Jozef Dodziuk. de Rham-Hodge theory for $L^{2}$-cohomology of infinite coverings. Topology, 16(2):157-165, 1977.

[DS02] Warren Dicks and Thomas Schick. The spectral measure of certain elements of the complex group ring of a wreath product. Geom. Dedicata, 93:121-137, 2002. 
[Eck00] Beno Eckmann. Introduction to $l_{2}$-methods in topology: reduced $l_{2}$-homology, harmonic chains, $l_{2}$-Betti numbers. Israel J. Math., 117:183-219, 2000. Notes prepared by Guido Mislin.

[ES05] Gábor Elek and Endre Szabó. Hyperlinearity, essentially free actions and $L^{2}$ invariants. The sofic property. Math. Ann., 332(2):421-441, 2005.

[Fol95] Gerald B. Folland. A course in abstract harmonic analysis. Studies in Advanced Mathematics. CRC Press, Boca Raton, FL, 1995.

[Gab02] Damien Gaboriau. Invariants $l^{2}$ de relations d'équivalence et de groupes. Publ. Math. Inst. Hautes Études Sci., (95):93-150, 2002.

[GLSŻ00] Rostislav I. Grigorchuk, Peter Linnell, Thomas Schick, and Andrzej Żuk. On a question of Atiyah. C. R. Acad. Sci. Paris Sér. I Math., 331(9):663-668, 2000.

[Gra10] Łukasz Grabowski. On the Atiyah problem for the lamplighter groups. ArXiv e-prints, September 2010. Available at http://arxiv.org/abs/1009.0229.

[GŻ01] Rostislav I. Grigorchuk and Andrzej Żuk. The lamplighter group as a group generated by a 2-state automaton, and its spectrum. Geom. Dedicata, 87(1-3):209-244, 2001.

[Hig61] Graham Higman. Subgroups of finitely presented groups. Proc. Roy. Soc. Ser. A, 262:455-475, 1961.

[Kec95] Alexander S. Kechris. Classical descriptive set theory, volume 156 of Graduate Texts in Mathematics. Springer-Verlag, New York, 1995.

[Lin93] Peter A. Linnell. Division rings and group von Neumann algebras. Forum Math., 5(6):561-576, 1993.

[LNW08] Franz Lehner, Markus Neuhauser, and Wolfgang Woess. On the spectrum of lamplighter groups and percolation clusters. Math. Ann., 342(1):69-89, 2008.

[LS01] Roger C. Lyndon and Paul E. Schupp. Combinatorial group theory. Classics in Mathematics. Springer-Verlag, Berlin, 2001. Reprint of the 1977 edition.

[LS07] Peter Linnell and Thomas Schick. Finite group extensions and the Atiyah conjecture. J. Amer. Math. Soc., 20(4):1003-1051 (electronic), 2007.

[Lüc94] Wolfgang Lück. Approximating $L^{2}$-invariants by their finite-dimensional analogues. Geom. Funct. Anal., 4(4):455-481, 1994.

[Lüc02] Wolfgang Lück. $L^{2}$-invariants: theory and applications to geometry and K-theory, volume 44 of Ergebnisse der Mathematik und ihrer Grenzgebiete. 3. Folge. A Series of Modern Surveys in Mathematics [Results in Mathematics and Related Areas. 3rd Series. A Series of Modern Surveys in Mathematics]. Springer-Verlag, Berlin, 2002.

[LW10] Franz Lehner and Stephan Wagner. Free Lamplighter Groups and a Question of Atiyah. ArXiv e-prints, May 2010. Available at http://arxiv.org/abs/1005.2347.

[PS Z10] Mikaël Pichot, Thomas Schick, and Andrzej Zuk. Closed manifolds with transcendental L2-Betti numbers. ArXiv e-prints, May 2010. Available at http://arxiv. org/abs/1005.1147.

[ST10] Roman Sauer and Andreas Thom. A spectral sequence to compute $L^{2}$-Betti numbers of groups and groupoids. J. Lond. Math. Soc. (2), 81(3):747-773, 2010.

[Tho08] Andreas Thom. Sofic groups and Diophantine approximation. Comm. Pure Appl. Math., 61(8):1155-1171, 2008. 